\title{
A CATALOG OF LOW SURFACE BRIGHTNESS GALAXIES. LIST II
}

\author{
JAMES M. SCHOMBERT \\ Infrared Processing and Analysis Center, Jet Propulsion Laboratory, California Institute of Technology, Pasadena, California 91125 \\ GREgory D. BOTHUN \\ Department of Physics, University of Oregon, Eugene, Oregon 97403 \\ STEPHEN E. Schneider \\ Department of Physics and Astronomy, University of Massachusetts, Amherst, Massachusetts 01002 \\ Stacy S. McGaugh \\ Department of Astronomy, University of Michigan, Ann Arbor, Michigan 48109 \\ Received 12 September 1991; revised 6 December 1991
}

\begin{abstract}
The second edition of a catalog of low surface brightness (LSB) galaxies is presented based on a visual search of the Second Palomar Sky Survey. This paper presents newly discovered LSB galaxies in 97 fields within the declination range of $0^{\circ}$ to $+25^{\circ}$. The primary catalog (objects with diameters greater than 1 arcmin) contains 198 objects; the secondary catalog (30 arcsec $<D<1$ arcmin) contains 140 objects. $\mathrm{H}$ I observations are presented for 171 of these newly discovered galaxies. Our $80 \%$ detection rate yields a fairly representative velocity distribution for a sample of LSB objects. The sample is dominated by LSB disks galaxies with $2000<v<9000 \mathrm{~km} \mathrm{~s}^{-1}$. Below $2000 \mathrm{~km} \mathrm{~s}^{-1}$ a few true dwarf galaxies are found. Approximately $10 \%$ of the sample has $v>10000 \mathrm{~km} \mathrm{~s}^{-1}$ and thus represents additional examples of truly large LSB galaxies. The $\mathrm{H}$ I redshift distribution is consistent with the hypothesis that LSB and HSB disk galaxies, in general, are drawn from the same distribution of physical size (e.g., disk scale length) modulo the curious tendency for the LSB sample to contain significantly more large-scale length galaxies which rival the prototype, Malin 1. Optical broadband and $\mathrm{H} \alpha$ imaging for a subset of the catalog is also presented confirming their quiescent nature. Our survey using the PSS-II is presently $35 \%$ complete and indicates that the UGC is $11.5 \%$ incomplete for a 1 mag arcsec ${ }^{-2}$ increase in surface brightness depth. Overall this survey demonstrates that LSB galaxies are not exclusively dwarf galaxies but encompass all Hubble types, although dominated by the late types, and all masses.
\end{abstract}

\section{INTRODUCTION}

A necessary requirement, in the study of the properties and evolution of galaxies, is that all types of galaxies must be discovered and represented in complete samples. In essence, this requires surveys which are optimized to detect particular classes of objects. In any such survey, however, the last class of objects which are detected and explored are those which have low contrast with respect to the background. In the optical window, this corresponds to the realm of objects whose mean surface brightness are buried in the background of the natural sky brightness. These objects, low surface brightness (LSB) galaxies, span the full range of Hubble types, although they are dominated by the extreme late types. Recovery of these objects is critical to our understanding of galaxy structure, formation, and evolution as well as to the very meaning of the Hubble sequence. These galaxies serve to offer us new windows into how the internal properties of a galaxy drive its morphological appearance. Moreover, if the mean surface brightness of a galaxy is related to the amplitude of the initial density perturbation from which it formed, then such objects may reveal a different pattern of large scale structure than has hitherto been detected via observations of high surface brightness (HSB) galaxies.

There seems to be a common misconception that the terms LSB galaxy and dwarf galaxy are interchangeable. One of the major aspects of this population that we hope to demonstrate is that, in fact, LSB galaxies are not exclusively low in mass (see also Bothun et al. 1990) and, furthermore,
LSB galaxies cover the same range in size and mass as high surface brightness (HSB) galaxies (see Sec. 4.2). The cataloging of LSB galaxies is also important to studies of star formation histories of galaxies in order to recover the full range of star formation efficiencies. This derives from the fact that, after excluding extinction or fading as the source of the low surface brightness, a galaxy with a low mean surface luminosity density implies an inefficient star formation process (few stars pc ${ }^{-3}$, Schombert et al. 1990).

Our survey for LSB galaxies is still in its infancy and is by no means a complete flux or surface brightness limited work. By comparison, a good catalog is one in which inclusion is based on some physical definition [e.g., an apparent magnitude limited catalog like the Revised Shapley-Ames catalog (Sandage \& Tammann 1981) or an angular diameter-limited catalog like the Uppsala General Catalog (Nilson 1973, hereafter referred to as UGC)] so that a completeness limit can be estimated. Any other sample is a list of objects, such as the Arp-Madore Atlas (Arp \& Madore 1987) where the galaxies were selected based on some morphological or general appearance criteria. Lists are, nonetheless, useful components of extragalactic studies by means of their ability to single out a particular realization of a physical phenomenon, such as starbursting galaxies. Within this context, the present catalog is clearly a list since the selection criteria are visual and no claim to completeness is made. This is particularly true since completeness in surface brightness is impossible to determine from ground-based observations. Our purpose with this work is simply to increase our known sam- 
ple of LSB galaxies and to offset the clear bias in apparent magnitude (as defined within regions brighter than 25 mag $\operatorname{arcsec}^{-2}$ ) catalogs for the discovery of only HSB systems. For, although LSB galaxies are low in contrast, they are by no means low in total magnitude (Bothun et al. 1986). Ultimately, our work on recovering LSB galaxies from the noise of the night sky is aimed at determining the space density of disk galaxies as a function of their central surface brightness. While selection effects are still important in this determination, without the discovery of these low contrast systems one could conclude that the canonical value of $B(0)=21.65 \pm 0.35 \mathrm{mag} \operatorname{arcsec}^{-2}$ (e.g., Freeman 1970) is correct and, that galaxies with $B(0)$ fainter than 23.0 mag $\operatorname{arcsec}^{-2}$ (e.g., $4 \sigma$ deviant) do not exist.

The plate material used herein is estimated from CCD data to be 1 mag arcsec ${ }^{-2}$ deeper than the plate material used for the UGC catalog (Schombert \& Bothun 1988). Incompleteness of the UGC was found to be $11.5 \%$ with this change in depth over a search area of $675^{\circ}$. This incompleteness is a combination of two factors: (1) Nilson, using a visual search, simply missed some LSB galaxies that would have met the angular diameter criterion and (2) some galaxies with diameters greater than one arcminute are just too low in visibility to have been detected on the original Sky Survey. For a sample of 109 spirals, Cornell et al. (1987) showed that the tabulated Nilson diameters correspond to an isophotal level of $25.36 \pm 0.71 \mathrm{mag} \operatorname{arcsec}^{-2}$. The vast majority of the galaxies contained in this sample and that of SB88 have isophotal diameters, at the 1 arcmin level, which are brighter than $26 \mathrm{mag} \operatorname{arcsec}^{-2}$ and thus should have been found by Nilson if the Cornell et al. (1987) sample is representative of the entire UGC catalog. This has been confirmed through inspection of the original Sky Survey where $90 \%$ of our galaxies can be recovered if you look hard enough. If LSB galaxies dominate the baryonic mass constituent of the universe, then one would have expected a much larger jump in the number of discovered objects depending on one's prejudice for $\Omega_{\text {baryon }}$. More substantial changes in the number of known LSB galaxies will require searches with specially treated plates (e.g., Malinization) or deep drift scan CCD surveys which allow "superflats" of $0.1 \%$ or better. However, searches by digital sky surveys will require computer algorithms to automatically detect LSB objects, which are difficult to implement. Visual searches are currently the only feasible method to obtain speed and sky coverage.

This paper presents the second portion of the LSB catalog confined to the declination zones $0^{\circ}-+25^{\circ}$ as mapped by the Second Palomar Sky Survey (PSS-II). Our first list (Schombert \& Bothun 1988, hereafter referred to as Paper I) was for the declination zone $+20^{\circ}$. However, in the interim, the PSS-II has proceeded gradually as constrained by weather and telescope operating conditions. Therefore, this second list contains random fields from these declination ranges. Section 2 describes the PSS-II and plate material plus procedures for identifying the LSB systems. Section 3 presents the catalog in its current state as well as $\mathrm{H}$ I observations. Section 4 discusses the morphological properties of LSB galaxies, the redshift distribution, $\mathrm{H}$ I masses, and optical imaging to date.

\section{OBSERVATIONS}

The observational material for this catalog comes solely from the blue plates of the Second Palomar Sky Survey
(Reid et al. 1991). The PSS-II is currently underway using the 48 in. Oschin Telescope at Palomar Observatory. The Oschin Telescope is a $f / 2.44$ Schmidt system and differs from the old 48 in. Schmidt of the original Sky survey in that it is now fixed with a new cemented doublet correcting lens (LLF6 and BK-7W). This new lens has improved the blue and near-UV transmission of the telescope and reduced image distortion on the new survey plates. The PSS-II is being performed in three bandpasses: $\mathrm{J}, \mathrm{F}$, and $\mathrm{N}$, corresponding to 3850-5500 $\AA, 6100-6900 \AA$, and 8000-9000 $\AA$, and with limiting magnitudes of $B_{J}<22.5, R_{c}<19.5$, and $I_{c}<19.5$ respectively (see Reid et al. 1991). Unlike the original Sky Survey, the spacing in the new Sky Survey is $5^{\circ}$ providing more overlap as an internal check to the completeness for any search program.

This catalog was produced from the $\mathrm{J}$ plates with the philosophy that the darker sky in the blue would facilitate detection of LSB objects plus the fact that the UGC was defined by blue plates from the old Survey. These plates are Kodak III $a$-J emulsions exposed for 60 minutes behind a GG 385 filter. The filter defines the blue edge of the bandpass whereas the emulsion sensitivity defines the red edge. All plates used for this study were "A" or "B" grade plates. Grade "A" plates are accepted for the final survey and have excellent depth and image quality. Grade " $B$ " plates have cosmetic defects which lower their quality for reproduction to the community, but do not degrade their scientific usage. Usually these defects were either numerous dust specks, aircraft and satellite trails or mild elongation of stellar images at the plate corners. A rigid criterion applied to all the plates used for this catalog is that their background densities must be between $0.9 D$ and $1.5 D$. This was to ensure a high enough and uniform background from which the LSB systems stand out in contrast. Every detection is also confirmed on a IIIa-F plate if available. About $90 \%$ of all discoveries exist on both plates (although much more difficult to discern on the $F$ plates), those which were not confirmed are marked as possibly unreal in the catalog notes unless neutral hydrogen was detected at $21 \mathrm{~cm}$.

The procedure for identifying new LSB galaxies from the plates is quite simple in that the search is carried out in a visual manner. The plate to be inspected is placed on a light table at an angle of $45^{\circ}$ (emulsion side down). One of us (JMS) would then scan the plate by eye from a distance of about $20 \mathrm{~cm}$ in a rasterlike pattern. Any object greater than 30 arcsec, and LSB in character, was marked. This procedure may appear crude at first, but several experiments have shown that, in fact, this is the fastest and most reliable method for detection. For example, machine scans use small pixels and, therefore, the value of the sky is dominated by noise introduced by grain size. This makes accurate detections of large, extended objects difficult. Visual inspection is also efficient in that during the scanning process the individual automatically rejects obvious plate defects and reflections from bright stars, thereby performing what is the equivalent of preliminary image analysis during the catalog search. We do note that Impey et al. (1992) have assembled a machineselected sample from the APM survey of 400 LSB objects located at equatorial declinations. A comparison of the properties of these two surveys should decide the relative efficacy of machine versus eye selection of LSB galaxies.

After identifying all LSB galaxies on a plate with a marker, PSS-I overlays are used to find which objects are undiscovered in the UGC. If the angular size is greater than 1 arc- 
min, and the object is missing from the UGC, then it is designated by the notation $\mathrm{FXXX}-\mathrm{N}$, where $\mathrm{XXX}$ is the field number of the Sky Survey and $N$ is a running number per field (the Sky Survey field numbers are the same as the UK Southern Sky Survey with inverse declination as found in UK Schmidt Southern Sky Survey). If the angular size is between $30 \mathrm{arcsec}$ and $1 \mathrm{arcmin}$, then the galaxy is designated FXXX-VN. The intent here is to identify all important LSB galaxies on a plate based on their contrast to sky brightness, then determine if the galaxy should be in the UGC or simply to be listed as interesting (the "V" class objects). After any other identification is noted (such as Zwicky number), the coordinates are determined by measurement from the nearest overlay grid lines and a low power $(7 \times)$ eyepiece is used to classify the galaxy. Paper I used nearby SAO stars for astrometry; however, this was a time-consuming task to achieve arcsecond accuracy of arcminute sized objects. For this paper, the coordinates are measured with a high-quality ruler good to $0.2 \mathrm{~mm}$. Internal and external checks shows that the positions herein are good to 15 arcsec, sufficient for $\mathrm{H}$ I beam observations or CCD imaging. Galaxy type was given following the standard Hubble system, as defined for LSB galaxies, in the Virgo cluster by Sandage \& Binggeli (1984). However, the extremely low contrast of many of the objects makes this process very subjective.

The observations of LSB galaxies for neutral hydrogen were begun in 1988 at Arecibo Observatory using the $315 \mathrm{~m}$ telescope. The observations were made using the standard total-power (position switching) mode with the $22 \mathrm{~cm}$ dualcircular polarization feed tuned to the center frequency of the range being observed. Each observation consisted typically of $5 \mathrm{~min}$ on-source $/ 5 \mathrm{~min}$ off-source integrations. The correlator was divided into four 512-channel, $20 \mathrm{MHz}$ segments, and each polarization was detected with two segments (overlapping by typically $2 \mathrm{MHz}$ ) to cover $\sim 7000$ $\mathrm{km} \mathrm{s}^{-1}$ in a single observation. After Hanning smoothing the resolution was about $16 \mathrm{~km} \mathrm{~s}^{-1}$, and the rms noise was typically better than $2 \mathrm{mJy}$. Basically, four velocity ranges were searched: low $\left(-500-6500 \mathrm{~km} \mathrm{~s}^{-1}\right)$, medium $(6500$ $13500 \mathrm{~km} \mathrm{~s}^{-1}$ ), high (13 500-20500 $\mathrm{km} \mathrm{s}^{-1}$ ), and extremely high (greater than $20500 \mathrm{~km} \mathrm{~s}^{-1}$ ). In our early observing programs and in crowded regions of the sky, we only had time to search the low and medium ranges, therefore, there is some probability that many of the nondetections are due to an unusually high redshift (see Sec. 4.2). The $\mathrm{H}$ I data herein are presented for the detections and the velocity search ranges for the nondetections. Nondetections were checked for correct coordinates by comparing stars in the finding charts with positions on the old sky survey prints. Several typos, transposed numbers and plain errors were found and the corrected coordinates for objects from Paper I are presented herein. The $\mathrm{H}_{\mathrm{I}}$ data reduction was all performed at Arecibo using a set of routines written by SES under the programming environment provided by the observatory's ANALYZ data reduction package. These routines automatically use information contained within the spectrum header to apply the calibration, correct for the zenith angle and frequency dependence of the gain, interactively fit a baseline to the spectrum and provide $F$-test statistics for the goodness of fit, plot the spectrum and print out other useful information from the spectrum's header, and finally store the reduced spectrum in a computer-readable format.

Optical imaging for a subsample of the LSB catalog was obtain from 1989 to 1991 at the McGraw-Hill $1.3 \mathrm{~m}$ tele- scope located at Michigan-Dartmouth-MIT Observatory on Kitt Peak mountain. The detector used was either a Thomson-CSF 400 by 576 CCD ( 0.48 arcsec per pixel) or a TI 4849584 by 390 CCD (0.46 arcsec per pixel). Broadband imaging used a CCD optimized Johnson $U B V R I$ set and $\mathrm{H} \alpha$ imaging used the Kitt Peak set No. 2. Exposure times were typically $600-900 \mathrm{~s}$ at Johnson $V$ and $R$. A full report on the photometry and structural properties will occur at a later date and the images are simply presented herein as a visual reference to the types of galaxies in this catalog and a guide to the appearance of star formation in LSB galaxies as mapped by the $\mathrm{H} \alpha$ images.

\section{THE CATALOG}

The primary catalog is presented in Table 1 where column 1 is the object name, column 2 is the coordinates (epoch 1950 ), column 3 is the angular diameter in arcminutes, column 4 is the the heliocentric velocity determined from the average of the velocities at $50 \%$ of peak intensity on each side of the $\mathrm{H}$ I profile, column 5 the integrated flux given in $\mathrm{Jy} \mathrm{km} \mathrm{s}^{-1}$ after correcting for frequency and zenith-angle dependence of the telescope gain, column 6 the width of the $\mathrm{H}$ I profile at $50 \%$ peak value, column 7 is the morphological type plus any comments, and column 8 is the search range in $\mathrm{km} \mathrm{s}^{-1}$ if no detection was obtained (see Sec. 3.2 for a more detailed description of the $\mathrm{H}$ I observations). If the object meets the UGC size requirement and, therefore, could be considered as missing from the UGC, then this is noted in the comments. The secondary catalog ("V" objects) is presented in Table 2 with the same format as Table 1 . The cata$\log$ is available by e-mail ( $j s @$ ipac.caltech.edu) or can be accessed from NED (NASA/IPAC Extragalactic Database, Helou et al. 1991). Unlike Paper I, where all objects greater than one arcminute are cataloged, only those objects which are clearly too low in surface brightness to have been included in the UGC are cataloged. There is no attempt to recover objects which, due to the deeper plate material, are simply larger than on the old survey prints and should be included in the UGC on this basis. On the other hand, a small HSB object with an extended LSB halo is included in this catalog regardless of whether the core had been identified in the CGCG.

The morphological classes are defined to conform as closely as possible to the Hubble sequence in the Virgo cluster (Sandage \& Binggeli 1984), since the Virgo cluster contains all known galaxy classes. However for clarity, and discussion of where LSB morphological deviations are important, the sequence used herein will be repeated below. The early type giant classes, E and S0, are rarely represented in LSB galaxies. Most early type galaxies of a LSB nature are the class of $\mathrm{D}$ and $\mathrm{cD}$ galaxies, distant ellipticals in rich clusters with extended envelopes (see F702-1), where the deeper plate material for the PSS-II reveals the previously undetected $\mathrm{cD}$ halo. There are also the occasional shell elliptical (Malin \& Carter 1980), whose LSB features were not visible on the old survey. There are several examples of LSB S0's ( see F571-7 and F611-2) noted for a distinct, HSB bulge, yet LSB disk.

The spiral classes, Sa to Sc, are handled in the same fashion as HSB Hubble types, using bulge to disk ratio and arm pitch as the primary criteria. If a ring is present then the notation $(r)$ is added to the classification (de Vaucouleurs 1959). Occasionally, a normal looking spiral (i.e., one with a 
TABLE 1. LSB catalog.

\begin{tabular}{|c|c|c|c|c|c|c|c|}
\hline Object & RA (1950) Dec & $\mathrm{D}$ & $V_{H I}$ & $S_{\nu}$ & $W_{50}$ & Notes & Search Range \\
\hline $\begin{array}{l}\text { F415-1 } \\
\text { F415-2 } \\
\text { F415-3 }\end{array}$ & $\begin{array}{l}0209.96+3234.8 \\
0216.88+2933.1 \\
0229.81+2837.1\end{array}$ & $\begin{array}{l}1.1 \\
1.2 \\
1.2\end{array}$ & 1018 & 12.52 & 72 & $\begin{array}{l}\mathrm{dE} / \mathrm{U} 1703 ? \\
\mathrm{E}_{\mathrm{p}} / \text { plumes } \\
\text { Sm/U2017? }\end{array}$ & $\begin{array}{c}-1421 \rightarrow 23500 \\
-642 \rightarrow 7365\end{array}$ \\
\hline $\begin{array}{l}\text { F469-1 } \\
\text { F469-2 }\end{array}$ & $\begin{array}{l}2252.79+3154.9 \\
2254.97+2742.9\end{array}$ & $\begin{array}{l}1.5 \\
1.0\end{array}$ & 2945 & 2.57 & 111 & $\begin{array}{l}\mathrm{E}_{\mathrm{p}} / \text { shells } \\
\mathrm{Sm}\end{array}$ & $-1553 \rightarrow 14248$ \\
\hline F473-1 & $0023.34+2338.6$ & 1.0 & 5632 & 0.44 & 111 & $\operatorname{Im}$ & \\
\hline $\begin{array}{l}\text { F477-1 } \\
\text { F477-2 }\end{array}$ & $\begin{array}{l}0151.78+2257.5 \\
0146.15+2543.5\end{array}$ & $\begin{array}{l}1.2 \\
1.1\end{array}$ & 4990 & 2.22 & 102 & $\begin{array}{l}\text { Im } \\
\text { S/ring/interaction? }\end{array}$ & $-642 \rightarrow 7365$ \\
\hline F480-1 & $0248.44+2622.5$ & 1.1 & 7485 & 2.24 & 142 & Sd & \\
\hline $\begin{array}{l}\text { F 495-1 } \\
\text { F495-2 }\end{array}$ & $\begin{array}{l}0813.77+2331.5 \\
0826.09+2702.1\end{array}$ & $\begin{array}{l}1.0 \\
1.0\end{array}$ & $\begin{array}{l}4269 \\
2162\end{array}$ & $\begin{array}{l}1.55 \\
1.25\end{array}$ & $\begin{array}{r}90 \\
115\end{array}$ & $\begin{array}{l}\text { S/Malin-like } \\
\text { Sd }\end{array}$ & \\
\hline F507-1 & $1531.44+2525.2$ & 1.0 & 10533 & 1.36 & 124 & Sc & \\
\hline $\begin{array}{l}\text { F508-1 } \\
\text { F508-2 } \\
\text { F508-3 } \\
\text { F508-4 }\end{array}$ & $\begin{array}{l}1300.53+2621.0 \\
1304.54+2300.5 \\
1315.73+2500.1 \\
1321.86+2538.9\end{array}$ & $\begin{array}{l}1.0 \\
1.5 \\
1.0 \\
1.0\end{array}$ & & & & $\begin{array}{l}\mathrm{dI} \\
\mathrm{Sb}_{\mathrm{p}} \\
\mathrm{Sd} \\
\mathrm{Sm} / \mathrm{Im}\end{array}$ & $9776 \rightarrow 18359$ \\
\hline F512-1 & $1431.13+2713.1$ & 1.0 & & & & $\mathrm{Sm}$ & \\
\hline F514-1 & $1517.87+2628.8$ & 1.0 & & & & $\mathrm{dI} / \mathrm{dE}$ & \\
\hline $\begin{array}{l}\text { F515-1 } \\
\text { F515-2 }\end{array}$ & $\begin{array}{l}1547.69+2644.9 \\
1556.24+2357.0\end{array}$ & $\begin{array}{l}1.0 \\
1.3\end{array}$ & & & & $\begin{array}{l}S(r) \\
S:\end{array}$ & $\begin{array}{l}-642 \rightarrow 32039 \\
-642 \rightarrow 32039\end{array}$ \\
\hline $\begin{array}{l}\text { F519-1 } \\
\text { F519-2 }\end{array}$ & $\begin{array}{l}1716.75+2629.0 \\
1720.52+2600.6\end{array}$ & $\begin{array}{l}1.0 \\
1.0\end{array}$ & & & & $\begin{array}{l}\mathrm{E} / \mathrm{S} 0 \\
\mathrm{Sc}\end{array}$ & \\
\hline $\begin{array}{l}\text { F520-1 } \\
\text { F520-2 } \\
\text { F520-3 }\end{array}$ & $\begin{array}{l}1726.86+2551.5 \\
1736.29+2600.8 \\
1746.68+2613.2\end{array}$ & $\begin{array}{l}1.0 \\
1.2 \\
1.0\end{array}$ & & & & $\begin{array}{l}\mathrm{SBb} \\
\mathrm{Im} / \mathrm{dI} \\
\mathrm{dI}\end{array}$ & \\
\hline $\begin{array}{l}\text { F530-1 } \\
\text { F530-2 } \\
\text { F530-3 }\end{array}$ & $\begin{array}{l}2105.36+2615.0 \\
2106.05+2701.8 \\
2118.71+2311.4\end{array}$ & $\begin{array}{l}1.0 \\
1.1 \\
1.0\end{array}$ & $\begin{array}{r}14339 \\
4769\end{array}$ & $\begin{array}{l}1.74 \\
2.83\end{array}$ & $\begin{array}{l}489 \\
169\end{array}$ & $\begin{array}{l}\text { S(r) } \\
\text { S(r) } \\
\text { Sc }\end{array}$ & $-642 \rightarrow 32039$ \\
\hline $\begin{array}{l}\text { F533-1 } \\
\text { F533-2 } \\
\text { F533-3 }\end{array}$ & $\begin{array}{l}2225.72+2306.7 \\
2231.56+2453.8 \\
2214.89+2457.8\end{array}$ & $\begin{array}{l}1.0 \\
1.2 \\
1.4\end{array}$ & $\begin{array}{r}1278 \\
12659\end{array}$ & $\begin{array}{l}2.37 \\
2.19\end{array}$ & $\begin{array}{r}58 \\
397\end{array}$ & $\begin{array}{l}\operatorname{Im} \\
\text { ring/Sb } \\
\mathrm{SBc}(\mathrm{r}) / \mathrm{LSB} \text { arms }\end{array}$ & $-642 \rightarrow 32039$ \\
\hline $\begin{array}{l}\text { F535-1 } \\
\text { F535-2 }\end{array}$ & $\begin{array}{l}2254.96+2743.0 \\
2305.86+2726.9\end{array}$ & $\begin{array}{l}1.0 \\
1.1\end{array}$ & & & & $\begin{array}{l}\mathrm{Sm} \\
\mathrm{SBm}\end{array}$ & \\
\hline $\begin{array}{l}\text { F539-1 } \\
\text { F539-2 }\end{array}$ & $\begin{array}{l}0014.75+2003.2 \\
0020.65+1959.6\end{array}$ & 1.0 & 5716 & 1.89 & 174 & $\begin{array}{l}\mathrm{Sc}(\mathrm{r}) \\
\mathrm{Im} / \text { lost U223? }\end{array}$ & $-642 \rightarrow 15275$ \\
\hline F542-1 & $0129.11+1820.3$ & 1.6 & & & & SB0/LSB arms & $-642 \rightarrow 23500$ \\
\hline $\begin{array}{l}\text { F544-1 } \\
\text { F544-2 }\end{array}$ & $\begin{array}{l}0158.59+1944.7 \\
0205.07+1845.0\end{array}$ & $\begin{array}{l}1.2 \\
1.0\end{array}$ & $\begin{array}{l}2336 \\
4368\end{array}$ & $\begin{array}{l}4.48 \\
3.88\end{array}$ & $\begin{array}{r}127 \\
87\end{array}$ & $\begin{array}{l}\mathrm{Sb} \\
\mathrm{Sm}\end{array}$ & \\
\hline F549-1 & $0359.27+2143.5$ & 1.1 & 6562 & 2.64 & 102 & $\mathrm{Sm}$ & \\
\hline F558-1 & $0659.71+2045.2$ & 1.0 & & & & $\mathrm{Sb}$ & $2961 \rightarrow 18359$ \\
\hline F558-2 & $0700.60+2015.0$ & 1.0 & & & & $\mathrm{Sb}$ & $-642 \rightarrow 23500$ \\
\hline
\end{tabular}


TABLE 1. (continued)

\begin{tabular}{|c|c|c|c|c|c|c|c|}
\hline Object & RA (1950) Dec & D & $V_{H I}$ & $S_{\nu}$ & $W_{50}$ & Notes & Search Range \\
\hline F560-2 & $0746.35+2154.2$ & 1.0 & & & & $\mathrm{E}_{\mathrm{p}} / \mathrm{LSB}$ halo & \\
\hline F560-3 & $0751.64+2011.5$ & 1.1 & & & & $\mathrm{Sb}$ & $1013 \rightarrow 15275$ \\
\hline F560-4 & $0743.41+1829.8$ & 1.1 & & & & $\mathrm{E}_{\mathrm{p}} /$ shells & $1013 \rightarrow 24528$ \\
\hline F560-5 & $0737.06+1721.9$ & 1.0 & 13080 & 1.46 & 263 & S/Malin-like & \\
\hline F561-1 & $0806.50+2242.4$ & 1.0 & & & & $\mathrm{Sm}$ & \\
\hline F561-2 & $0812.45+2142.7$ & 1.2 & 4276 & 1.70 & 280 & $\mathrm{Sb}$ & \\
\hline F561-3 & $0810.04+1947.2$ & 1.0 & & & & $\mathrm{~S} 0 / \mathrm{Sa}$ & $1013 \rightarrow 23500$ \\
\hline F563-1 & $0852.17+1956.6$ & 1.0 & & & & $\mathrm{Sm} / \mathrm{Im}$ & \\
\hline F564-1 & $0907.67+2045.7$ & 1.5 & 8509 & 1.52 & 502 & $\mathrm{SBb}_{\mathrm{p}}$ & \\
\hline F564-2 & $0903.21+1715.2$ & 1.2 & 16970 & 1.71 & 499 & $\mathrm{Sc}(\mathrm{r}) / \mathrm{LSB}$ arms & \\
\hline F565-1 & $0919.74+2104.0$ & 1.1 & 9486 & 1.70 & 339 & $\mathrm{Sb}$ & \\
\hline F567-1 & $1016.27+2132.0$ & 1.2 & 1091 & 0.79 & 45 & $\mathrm{E}_{\mathrm{p}} /$ interaction? & \\
\hline F567-2 & $1015.19+2118.8$ & 1.3 & 5691 & 1.07 & 56 & $\mathrm{Sm}$ & \\
\hline F568-1 & $1023.38+2241.0$ & 1.0 & 6526 & 1.73 & 124 & $\mathrm{Sc}$ & \\
\hline F568-3 & $1024.25+2228.9$ & 1.0 & 5908 & 1.69 & 157 & $\mathrm{Sd}$ & \\
\hline F568-5 & $1027.85+2206.7$ & 1.1 & 6523 & 0.92 & 281 & $\mathrm{Sb}_{\mathrm{p}}$ & \\
\hline F568-6 & $1037.14+2106.4$ & 1.9 & 13827 & 3.49 & 386 & S/Malin-like & \\
\hline F568-7 & $1026.76+2101.7$ & 1.1 & & & & $\mathrm{Sc}_{\mathrm{p}}$ & \\
\hline F568-8 & $1031.42+1957.9$ & 1.1 & & & & $\mathrm{Sb}$ & $2961 \rightarrow 23500$ \\
\hline F568-9 & $1025.48+1851.9$ & 1.5 & 8068 & 2.35 & 333 & $\mathrm{SBc}_{\mathrm{p}}$ & \\
\hline F570-1 & $1115.32+2246.6$ & 1.1 & & & & SB0: & $1013 \rightarrow 14248$ \\
\hline F570-3 & $1120.36+1945.1$ & 1.0 & & & & $\mathrm{dI} / \mathrm{dE}$ & $-642 \rightarrow 91111$ \\
\hline F570-4 & $1110.30+1902.2$ & 1.0 & & & & $\mathrm{~S} 0 / \mathrm{Sa}$ & $5882 \rightarrow 21957$ \\
\hline F570-5 & $1122.68+1804.9$ & 1.1 & 4921 & 4.22 & 302 & So & \\
\hline F570-6 & $1115.04+1805.6$ & 1.2 & & & & $\mathrm{SB} 0_{\mathrm{p}}$ & $1013 \rightarrow 9111$ \\
\hline F570-7 & $1115.76+1758.7$ & 1.0 & 1051 & 1.54 & 59 & $\mathrm{dI}$ & \\
\hline F571-2 & $1142.69+2142.0$ & 1.0 & & & & $S c_{p}$ & $2961 \rightarrow 17331$ \\
\hline F571-4 & $1123.67+2120.0$ & 1.1 & & & & $\mathrm{Sc}(\mathrm{r}) / \mathrm{LSB}$ arms & $1013 \rightarrow 14248$ \\
\hline F571-5 & $1127.76+2051.6$ & 1.0 & 4253 & 2.30 & 130 & $\mathrm{Sm}$ & \\
\hline F571-6 & $1128.48+2045.3$ & 1.1 & 6157 & 2.68 & 101 & $\operatorname{Irr}_{p}$ & \\
\hline F571-7 & $1136.09+2000.5$ & 1.2 & & & & So & . \\
\hline F571-8 & $1131.30+1937.8$ & 1.5 & 3761 & 2.04 & 295 & $\mathrm{Sb}$ & \\
\hline F571-10 & $1126.20+1833.4$ & 1.2 & & & & $\mathrm{dE}$ & $-1553 \rightarrow 9111$ \\
\hline F572-2 & $1153.18+1810.3$ & 1.1 & 6346 & 3.79 & 313 & $S c_{p}$ & \\
\hline F573-1 & $1225.58+2234.2$ & 1.0 & & & & $\mathrm{dI}$ & $452 \rightarrow 9111$ \\
\hline F573-2 & $1216.55+2134.5$ & 1.0 & 8754 & 1.25 & 284 & $\mathrm{Sb}$ & \\
\hline F573-3 & $1207.14+2019.6$ & 1.0 & 2494 & 1.31 & 71 & Sd & \\
\hline F573-4 & $1215.72+2017.7$ & 1.0 & & & & $\mathrm{Sa}$ & \\
\hline F573-5 & $1214.68+1921.7$ & 1.1 & & & & $\mathrm{~S}_{\mathrm{p}}$ & $2961 \rightarrow 17331$ \\
\hline F573-6 & $1225.14+1842.6$ & 1.0 & & & & $\mathrm{dEn}$ & \\
\hline F573-7 & $1213.77+1804.6$ & 1.0 & 8883 & 0.59 & 138 & $\mathrm{Sc}$ & \\
\hline F573-9 & $1227.67+1740.8$ & 1.1 & & & & Sd & \\
\hline F573-10 & $1219.87+1718.7$ & 1.1 & & & & $\mathrm{dE}$ & $-642 \rightarrow 23500$ \\
\hline F574-1 & $1235.63+2235.3$ & 1.0 & 6890 & 1.63 & 209 & Sd & \\
\hline F574-2 & $1244.23+2206.1$ & 1.0 & 6319 & 0.69 & 48 & Sm: & \\
\hline
\end{tabular}




\begin{tabular}{|c|c|c|c|c|c|c|c|}
\hline Object & RA (1950) Dec & $\mathrm{D}$ & $V_{H I}$ & $S_{\nu}$ & $W_{50}$ & Notes & Search Range \\
\hline F574-3 & $1225.64+2027.2$ & 1.2 & 6777 & 3.20 & 181 & $\mathrm{Sa}$ & \\
\hline F574-5 & $1226.10+1945.1$ & 1.3 & 13548 & 1.12 & 252 & $\mathrm{Sb}_{\mathrm{p}}$ & \\
\hline F574-6 & $1245.84+1854.4$ & 1.0 & & & & dI & $-1060 \rightarrow 14248$ \\
\hline F574-7 & $1237.01+1838.6$ & 1.0 & 6679 & 0.34 & 120 & S0 & \\
\hline F574-8 & $1230.15+1817.3$ & 1.1 & & & & So & \\
\hline F574-9 & $1239.84+1746.9$ & 1.1 & & & & $\mathrm{SO}_{\mathrm{p}}$ & $2961 \rightarrow 11165$ \\
\hline F574-10 & $1248.03+1744.4$ & 1.2 & 863 & 5.58 & 81 & $\mathrm{Sd} / \mathrm{Sm}$ & \\
\hline F574-11 & $1235.84+1949.4$ & 1.1 & 1363 & 4.36 & 72 & $\mathrm{Sd} / \mathrm{Irr}$ & \\
\hline F575-1 & $1300.27+2215.0$ & 1.0 & & & & $\mathrm{dE}$ & $-2883 \rightarrow 10138$ \\
\hline F575-2 & $1249.32+2200.7$ & 1.0 & 583 & 1.56 & 30 & $\mathrm{Sm} / \mathrm{Irr}$ & \\
\hline F575-3 & $1253.24+1929.6$ & 1.2 & 419 & 3.45 & 23 & & \\
\hline F575-4 & $1302.03+1801.6$ & 1.2 & & & & $\mathrm{dE}$ & $-934 \rightarrow 9111$ \\
\hline F575-5 & $1.307 .49+1725.9$ & 1.5 & 3584 & 3.00 & 140 & $\mathrm{Sd} / \mathrm{Irr}$ & \\
\hline F576-1 & $1311.26+2253.3$ & 1.4 & 3235 & 2.56 & 238 & $\mathrm{Sc}$ & \\
\hline F576-3 & $1308.27+2138.1$ & 1.0 & 6750 & 1.28 & 224 & Sd & \\
\hline F577-1 & $1341.15+1818.5$ & 1.3 & & & & $\mathrm{E}_{\mathrm{p}}$ & $1013 \rightarrow 15275$ \\
\hline F577-2 & $1349.87+1732.5$ & 1.1 & 7712 & 2.99 & 341 & $\mathrm{Sb}$ & \\
\hline F578-1 & $1410.32+2037.3$ & 1.0 & & & & $\mathrm{E} / \mathrm{S} 0$ & $1013 \rightarrow 11165$ \\
\hline F578-2 & $1351.90+1940.0$ & 1.1 & 8230 & 1.83 & 150 & $\mathrm{Sc}$ & \\
\hline F579-1 & $1409.48+2136.9$ & 1.0 & 8477 & 2.37 & 375 & $\mathrm{Sb}$ & \\
\hline F579-2 & $1412.63+2115.5$ & 1.0 & 8668 & 1.23 & 231 & Sc: & \\
\hline F579-3 & $1411.61+2112.7$ & 1.0 & 8538 & 1.44 & 134 & $\mathrm{SBc}_{\mathrm{p}}$ & \\
\hline F579-4 & $1431.43+1905.0$ & 1.0 & & & & $\mathrm{dI}:$ & $1013 \rightarrow 15275$ \\
\hline F580-2 & $1434.73+2107.6$ & 1.4 & & & & $\mathrm{Sc}(\mathrm{r})$ & $2961 \rightarrow 17331$ \\
\hline F580-3 & $1439.02+1918.8$ & 1.7 & & & & $\mathrm{Sb}(\mathrm{r})$ & \\
\hline F580-6 & $1437.70+2148.7$ & 1.2 & & & & $\mathrm{Sc}_{\mathrm{p}}$ & $1013 \rightarrow 15275$ \\
\hline F581-2 & $1454.56+2007.5$ & 1.1 & & & & $\mathrm{SO}_{\mathrm{p}}$ & $1013 \rightarrow 15275$ \\
\hline F582-1 & $1528.43+2302.6$ & 1.0 & & & & $\mathrm{Sb}$ & $1013 \rightarrow 15275$ \\
\hline F582-2 & $1531.76+2157.2$ & 1.5 & 7043 & 4.71 & 283 & $\mathrm{Sc}$ & \\
\hline F582-3 & $1535.02+2002.8$ & 1.0 & 10602 & 0.47 & 161 & Sc & \\
\hline F583-1 & $1555.28+2048.4$ & 1.0 & 2264 & 7.04 & 162 & $\mathrm{Sm} / \mathrm{Irr}$ & \\
\hline F583-2 & $1534.79+2057.5$ & 1.4 & 1720 & 4.61 & 84 & $\mathrm{Sd} / \mathrm{Sm}$ & \\
\hline F583-3 & $1549.35+2021.0$ & 1.0 & & & & $\mathrm{SO}_{\mathrm{p}}$ & \\
\hline F583-4 & $1549.97+1856.0$ & 1.0 & 3611 & 1.37 & 115 & Sc & \\
\hline F583-5 & $1543.47+1727.9$ & 1.9 & & & & $\mathrm{Sb}$ & \\
\hline F584-1 & $1603.46+2219.3$ & 1.0 & 11981 & 0.93 & 326 & $\mathrm{Sb}$ & \\
\hline F585-1 & $1630.92+2254.0$ & 1.0 & & & & $\mathrm{E} / \mathrm{S} 0$ & $2961 \rightarrow 11165$ \\
\hline F585-3 & $1619.26+2058.9$ & 1.1 & & & & $\mathrm{Sm}$ & \\
\hline F597-1 & $2041.71+2153.1$ & 1.1 & & & & Sc & $-642 \rightarrow 32039$ \\
\hline F597-2 & $2041.99+1832.9$ & 1.0 & 6880 & 2.77 & 204 & $\mathrm{SBc}$ & \\
\hline F602-1 & $2232.36+2218.1$ & 1.2 & 7608 & 1.86 & 41 & Sc & \\
\hline F608-1 & $0014.71+1715.3$ & 1.0 & 1031 & 2.02 & 75 & $\mathrm{Im} / \mathrm{Sm} / \mathrm{UGC} 159 ?$ & \\
\hline F611-1 & $0117.12+1631.7$ & 1.0 & 2166 & 1.52 & 28 & $\mathrm{dI} / \mathrm{Im}$ & \\
\hline F611-2 & $0111.63+1510.0$ & 1.6 & 11306 & 2.78 & 372 & E/S0/shells & \\
\hline
\end{tabular}


TABLE 1. (continued)

\begin{tabular}{|c|c|c|c|c|c|c|c|}
\hline Object & RA (1950) Dec & $\mathrm{D}$ & $V_{H I}$ & $S_{\nu}$ & $W_{50}$ & Notes & Search Range \\
\hline F611-3 & $0110.68+1427.7$ & 1.0 & 13010 & 1.10 & 313 & $\mathrm{Sc}(\mathrm{r}) / \mathrm{LSB}$ disk & \\
\hline F612-1 & $0127.55+1425.5$ & 1.6 & 2448 & 3.79 & 125 & $\mathrm{Sm}$ & \\
\hline F614-1 & $0212.98+1649.6$ & 1.0 & 8236 & 1.43 & 103 & $\mathrm{Sm}$ & \\
\hline $\begin{array}{l}\text { F615-1 } \\
\text { F615-2 }\end{array}$ & $\begin{array}{l}0240.63+1631.5 \\
0244.17+1439.3\end{array}$ & $\begin{array}{l}1.2 \\
1.4\end{array}$ & $\begin{array}{r}818 \\
7596\end{array}$ & $\begin{array}{l}1.60 \\
1.23\end{array}$ & $\begin{array}{r}35 \\
119\end{array}$ & $\begin{array}{l}\mathrm{dI} \\
\mathrm{Sc}(\mathrm{r}) / \mathrm{LSB} \text { arms }\end{array}$ & \\
\hline $\begin{array}{l}\text { F620-1 } \\
\text { F620-2 } \\
\text { F } 620-3\end{array}$ & $\begin{array}{l}0415.02+1317.5 \\
0422.23+1242.8 \\
0428.70+1720.3\end{array}$ & $\begin{array}{l}1.7 \\
1.2 \\
1.0\end{array}$ & & & & $\begin{array}{l}\mathrm{Sc}(\mathrm{r}) \\
\mathrm{Sc} \\
\mathrm{dEn}:\end{array}$ & \\
\hline F633-1 & $0845.67+1622.9$ & 1.0 & & & & $\mathrm{Sc}$ & \\
\hline $\begin{array}{l}\text { F635-1 } \\
\text { F635-2 }\end{array}$ & $\begin{array}{l}0919.78+1333.6 \\
0927.38+1411.7\end{array}$ & $\begin{array}{l}1.4 \\
1.5\end{array}$ & & & & $\begin{array}{l}\text { E/LSB halo } \\
\text { Irr }\end{array}$ & \\
\hline F636-1 & $0932.48+1720.5$ & 1.2 & 4302 & 2.43 & 75 & $\mathrm{Sm}$ & \\
\hline $\begin{array}{l}\text { F637-1 } \\
\text { F637-2 } \\
\text { F637-3 }\end{array}$ & $\begin{array}{l}0953.36+1645.2 \\
0955.08+1359.3 \\
0958.12+1739.0\end{array}$ & $\begin{array}{l}1.0 \\
1.3 \\
1.0\end{array}$ & & & & $\begin{array}{l}\mathrm{SBc} / \mathrm{E} \text { comp/triplet? } \\
\mathrm{dI} \\
\mathrm{Sb}(\mathrm{r})\end{array}$ & \\
\hline $\begin{array}{l}\text { F638-1 } \\
\text { F638-2 } \\
\text { F638-3 } \\
\text { F638-4 }\end{array}$ & $\begin{array}{l}1015.70+1331.8 \\
1019.20+1411.7 \\
1029.84+1454.4 \\
1032.08+1629.4\end{array}$ & $\begin{array}{l}2.0 \\
1.0 \\
1.2 \\
1.0\end{array}$ & $\begin{array}{l}5471 \\
8613 \\
3160 \\
5856\end{array}$ & $\begin{array}{l}1.77 \\
1.10 \\
1.81 \\
1.15\end{array}$ & $\begin{array}{r}57 \\
292 \\
42 \\
33\end{array}$ & $\begin{array}{l}\mathrm{Sc} \\
\mathrm{Sb}_{\mathrm{p}} \\
\mathrm{Sm} \\
\mathrm{Sm}:\end{array}$ & \\
\hline $\begin{array}{l}\text { F640-1 } \\
\text { F640-2 } \\
\text { F640-3 }\end{array}$ & $\begin{array}{l}1056.24+1424.0 \\
1100.80+1617.2 \\
1109.64+1701.6\end{array}$ & $\begin{array}{l}1.1 \\
1.0 \\
1.3\end{array}$ & 1227 & 0.95 & 92 & $\begin{array}{l}\text { S/Malin-like } \\
\text { S/smooth } \\
\text { dI/dE }\end{array}$ & $\begin{array}{l}-642 \rightarrow 23500 \\
-642 \rightarrow 23500\end{array}$ \\
\hline $\begin{array}{l}\text { F644-3 } \\
\text { F644-4 } \\
\text { F644-5 }\end{array}$ & $\begin{array}{l}1211.63+1614.0 \\
1223.05+1607.4 \\
1216.99+1415.0\end{array}$ & $\begin{array}{l}1.0 \\
1.1 \\
1.0\end{array}$ & & & & $\begin{array}{l}\mathrm{dI} \\
\mathrm{Im} \\
\mathrm{dI}\end{array}$ & $\begin{array}{l}-642 \rightarrow 15275 \\
-642 \rightarrow 15275\end{array}$ \\
\hline $\begin{array}{l}\text { F646-1 } \\
\text { F646-2 } \\
\text { F646-3 }\end{array}$ & $\begin{array}{l}1250.47+1440.2 \\
1253.92+1521.2 \\
1307.45+1726.0\end{array}$ & $\begin{array}{l}1.1 \\
1.2 \\
1.1\end{array}$ & & & & $\begin{array}{l}\text { Sm/Irr (Rea 21) } \\
\text { dE (Rea 66) } \\
\text { S/ring }\end{array}$ & \\
\hline F650-1 & $1413.95+1406.4$ & 1.6 & & & & $\mathrm{dI}: /$ real? & $-642 \rightarrow 15275$ \\
\hline F651-1 & $1435.20+1331.9$ & 1.0 & 6576 & 1.62 & 133 & Sd & \\
\hline $\mathrm{F} 651-2$ & $1444.02+1425.6$ & 1.4 & 1789 & 4.01 & 50 & $\mathrm{dI} / \mathrm{Im}$ & \\
\hline $\begin{array}{l}\text { F657-1 } \\
\text { F657-2 }\end{array}$ & $\begin{array}{l}1635.96+1422.4 \\
1645.66+1532.1\end{array}$ & $\begin{array}{l}1.5 \\
1.0\end{array}$ & & & & $\begin{array}{l}\text { Irr } \\
\text { Sc }\end{array}$ & $-642 \rightarrow 7365$ \\
\hline $\begin{array}{l}\text { F673-1 } \\
\text { F673-2 }\end{array}$ & $\begin{array}{l}2150.05+1533.2 \\
2212.11+1331.4\end{array}$ & $\begin{array}{l}1.0 \\
1.1\end{array}$ & 7333 & 2.69 & 249 & $\begin{array}{l}\text { Sb } \\
\text { S/Malin-like }\end{array}$ & $-642 \rightarrow 32039$ \\
\hline F674-1 & $2216.72+1446.9$ & 1.0 & 6874 & 1.43 & 210 & $\mathrm{Sm}$ & \\
\hline F675-1 & $2238.12+1342.8$ & 1.0 & & & & S/ring & $-642 \rightarrow 32039$ \\
\hline $\begin{array}{l}\text { F677-1 } \\
\text { F677-2 } \\
\text { F677-3 }\end{array}$ & $\begin{array}{l}2309.48+1533.6 \\
2316.63+1527.3 \\
2319.25+1631.1\end{array}$ & $\begin{array}{l}1.0 \\
1.1 \\
1.0\end{array}$ & 7149 & 1.63 & 109 & $\begin{array}{l}\text { Sm } \\
\text { Irr } \\
\text { Sm }\end{array}$ & $\begin{array}{l}-642 \rightarrow 32039 \\
-642 \rightarrow 15275\end{array}$ \\
\hline F678-1 & $2334.24+1720.7$ & 1.0 & 4184 & 3.72 & 137 & Irr & \\
\hline F682-1 & $0054.92+1005.6$ & 1.0 & 2756 & 2.37 & 114 & $\mathrm{Sm}$ & \\
\hline
\end{tabular}


TABLE 1. (continued)

\begin{tabular}{|c|c|c|c|c|c|c|c|}
\hline Object & RA (1950) Dec & $\mathrm{D}$ & $V_{H I}$ & $S_{\nu}$ & $W_{50}$ & Notes & Search Range \\
\hline F683-1 & $0119.92+0829.9$ & 1.3 & & & & $\mathrm{dI}: /$ real?/N524 cluster & $-642 \rightarrow 15275$ \\
\hline $\begin{array}{l}\text { F687-1 } \\
\text { F687-2 }\end{array}$ & $\begin{array}{l}0237.71+1043.9 \\
0243.43+0813.7\end{array}$ & $\begin{array}{l}1.0 \\
1.0\end{array}$ & $\begin{array}{l}3662 \\
7634\end{array}$ & $\begin{array}{l}2.79 \\
1.57\end{array}$ & $\begin{array}{r}115 \\
84\end{array}$ & $\begin{array}{l}\text { Sm: } \\
\text { S(r)/Malin-like }\end{array}$ & \\
\hline $\begin{array}{l}\text { F702-1 } \\
\text { F702-2 }\end{array}$ & $\begin{array}{l}0739.73+0929.3 \\
0741.45+0921.2\end{array}$ & $\begin{array}{l}1.5 \\
1.1\end{array}$ & & & & $\begin{array}{l}\text { E/cD (NGC2433?) } \\
\mathrm{E}_{\mathrm{p}} / \mathrm{LSB} \text { halo }\end{array}$ & \\
\hline F704-1 & $0820.79+1012.6$ & 1.0 & 4237 & 2.50 & 164 & $\mathrm{SBm}(\mathrm{r})$ & \\
\hline F709-1 & $0950.31+0815.8$ & 1.1 & 2640 & 3.34 & 121 & Sd & \\
\hline F721-1 & $1400.77+0959.6$ & 1.2 & 7210 & 1.52 & 64 & $\mathrm{Sm}$ & \\
\hline $\begin{array}{l}\text { F723-1 } \\
\text { F723-2 }\end{array}$ & $\begin{array}{l}1432.92+0943.3 \\
1450.05+1023.9\end{array}$ & $\begin{array}{l}1.1 \\
1.0\end{array}$ & & & & $\begin{array}{l}\mathrm{Sa} \\
\mathrm{SBm}\end{array}$ & \\
\hline F727-1 & $1559.95+1224.8$ & 1.0 & & & & $\mathrm{Sc}$ & \\
\hline $\begin{array}{l}\text { F728-1 } \\
\text { F728-2 }\end{array}$ & $\begin{array}{l}1621.42+1129.3 \\
1626.67+1129.3\end{array}$ & $\begin{array}{l}1.0 \\
1.0\end{array}$ & 4977 & 2.04 & 125 & $\begin{array}{l}\text { Sc/near N6132 } \\
\text { Sm/ring }\end{array}$ & $-642 \rightarrow 7365$ \\
\hline F730-1 & $1654.21+0851.0$ & 1.0 & & & & $\mathrm{dI} / \mathrm{dE}$ & $-642 \rightarrow 7365$ \\
\hline F740-1 & $2017.38+0928.6$ & 1.1 & 5493 & 1.54 & 149 & $\mathrm{Sm}$ & \\
\hline F743-1 & $2116.30+0809.2$ & 1.0 & 2959 & 2.60 & 130 & Sd & \\
\hline F744-1 & $2145.19+1157.2$ & 1.0 & 4663 & 2.96 & 119 & Sd & \\
\hline $\begin{array}{l}\text { F746-1 } \\
\text { F746-2 } \\
\text { F746-3 }\end{array}$ & $\begin{array}{l}2218.38+1234.9 \\
2221.65+0826.5 \\
2226.07+0756.5\end{array}$ & $\begin{array}{l}1.0 \\
1.0 \\
1.1\end{array}$ & $\begin{array}{r}7850 \\
12040 \\
7621\end{array}$ & $\begin{array}{l}2.59 \\
1.53 \\
1.40\end{array}$ & $\begin{array}{l}140 \\
102 \\
151\end{array}$ & $\begin{array}{l}\text { Irr } \\
\text { S/Malin-like } \\
\text { Irr }\end{array}$ & \\
\hline $\begin{array}{l}\text { F750-1 } \\
\text { F750-2 }\end{array}$ & $\begin{array}{l}2335.30+1102.4 \\
2337.61+0702.0\end{array}$ & $\begin{array}{l}1.1 \\
1.1\end{array}$ & 3713 & 2.71 & 94 & $\begin{array}{l}\mathrm{Sc} \\
\mathrm{Sc}\end{array}$ & $-642 \rightarrow 32039$ \\
\hline F $750-3$ & $2339.69+1048.0$ & 1.0 & & & & $\mathrm{Sb}$ & $-642 \rightarrow 15275$ \\
\hline F750-4 & $2341.84+0950.6$ & 1.2 & 1504 & 2.44 & 118 & $\mathrm{Sc}(\mathrm{r})$ & \\
\hline F750-5 & $2343.03+1118.1$ & 1.5 & 5367 & 4.17 & 179 & $\mathrm{Sc}(\mathrm{r})$ & \\
\hline F $821-1$ & $2319.26+0333.1$ & 1.0 & & & & $\mathrm{Sc}_{\mathrm{p}} /$ on & $-1908 \rightarrow 6030$ \\
\hline F821-2 & $2320.97+024636$ & 1.1 & & & & & $1013 \rightarrow 9111$ \\
\hline
\end{tabular}

HSB disk ) has unusually extended LSB arms, differing from a simpler LSB disk and bulge. If so, this is also noted in the catalog. The Sd class is reserved for those LSB galaxies with a discernible spiral pattern, yet no bulge (integral sign galaxies). The Sm class is defined by a weak, fragmented spiral pattern and no bulge. The tail end of the late type galaxies is represented by classes Im and Irr. The Im types are similar to the Virgo Im's, having a very low surface-brightness halo embedded with low contrast lumps in no discernible spiral pattern. The Irr types are similar to the Im, but here the lumps are much higher in surface brightness, often with some central concentration, intermixed in a LSB halo.

Parallel to the giant elliptical and spiral sequence are the dwarf galaxy classes. In this paper, the dwarfs are divided into two types based solely on smoothness and isophotal shape, dE's and dI's. Both types are defined by their very low surface brightness, lack of any strong central concentration and brightness profiles which are very nearly flat, rather than power law as with ellipticals or spiral bulges. dI's are separated from dE's in that their isophotes are irregular in shape with asymmetric regions of higher surface brightness.
dE's have smooth profiles well described by ellipses. dE's with stellar nuclei are marked dEn.

Outside the Hubble sequence, there are the peculiar and ring systems, described extensively by the Arp-Madore Atlas (Arp \& Madore 1987). An attempt is made here to avoid filling the catalog with HSB galaxies which exhibit LSB tidal features. As noted in Paper I, the number of galaxies which become "peculiar" in the sense of exhibiting LSB tails, plumes, and shells increases rapidly as deeper plate material is used. The galaxies in this catalog are selected to be primarily LSB in nature, rather than a HSB galaxy with a LSB feature that only embodies a small percentage of the total mass. Thus, the peculiar galaxies in this catalog are in general LSB in nature and are classified as peculiar because they fail to conform to any known Hubble type. Any galaxy which has a peculiar feature, such a distorted disk or arm is flagged with a subscript " $p$." The ring types are self-explanatory; however, an unusually large occurrence $(4.5 \%)$ is found in this sample (their detection in $\mathrm{H}$ I precludes them from being galactic objects).

Lastly, we introduce a new class of LSB galaxies in an 
TABLE 2. VLSB catalog.

\begin{tabular}{|c|c|c|c|c|c|c|c|}
\hline Object & RA (1950) Dec & $\mathrm{D}$ & $V_{H I}$ & $S_{\nu}$ & $W_{50}$ & Notes & Search Range \\
\hline $\begin{array}{l}\text { F473-V1 } \\
\text { F473-V2 } \\
\text { F473-V3 }\end{array}$ & $\begin{array}{l}0014.71+2635.0 \\
0023.73+2422.0 \\
0032.15+2230.2\end{array}$ & $\begin{array}{l}0.6 \\
0.8 \\
0.5\end{array}$ & $\begin{array}{l}3571 \\
5835\end{array}$ & $\begin{array}{l}1.35 \\
1.11\end{array}$ & $\begin{array}{r}94 \\
143\end{array}$ & $\begin{array}{l}\operatorname{Im} \\
\operatorname{Irr} \\
\text { Sm }\end{array}$ & \\
\hline $\begin{array}{l}\text { F477-V1 } \\
\text { F477-V2 }\end{array}$ & $\begin{array}{l}0156.48+2646.1 \\
0151.73+2237.5\end{array}$ & $\begin{array}{l}0.7 \\
0.5\end{array}$ & $\begin{array}{l}5309 \\
5039\end{array}$ & $\begin{array}{l}1.74 \\
1.00\end{array}$ & $\begin{array}{r}136 \\
62\end{array}$ & $\begin{array}{l}\mathrm{dI} \\
\mathrm{dI}\end{array}$ & \\
\hline $\begin{array}{l}\text { F480-V1 } \\
\text { F480-V2 } \\
\text { F480-V3 } \\
\text { F480-V4 } \\
\text { F480-V5 } \\
\text { F480-V6 } \\
\text { F480-V7 }\end{array}$ & $\begin{array}{l}0246.91+2627.1 \\
0248.91+2545.2 \\
0251.82+2310.5 \\
0259.95+2548.3 \\
0300.35+2719.2 \\
0300.68+2715.5 \\
0305.39+2704.5\end{array}$ & $\begin{array}{l}0.7 \\
0.8 \\
0.8 \\
0.9 \\
0.7 \\
0.5 \\
0.6\end{array}$ & $\begin{array}{r}6073 \\
10619\end{array}$ & $\begin{array}{l}1.60 \\
1.47\end{array}$ & $\begin{array}{l}100 \\
118\end{array}$ & $\begin{array}{l}\text { Sm/Im } \\
\text { Sb/distant? } \\
\text { SBm } \\
\text { Sb } \\
\text { S: } \\
\text { S:/ring } \\
\text { S/Irr }\end{array}$ & $\begin{array}{l}-642 \rightarrow 32039 \\
-642 \rightarrow 32039\end{array}$ \\
\hline F495-V1 & $0825.29+2557.4$ & 0.7 & 2269 & 0.45 & 46 & $\mathrm{dI}$ & \\
\hline F500-V1 & $1020.79+2302.7$ & 0.8 & & & & $\mathrm{Sm}$ & \\
\hline $\begin{array}{l}\text { F507-V1 } \\
\text { F507-V2 } \\
\text { F507-V3 }\end{array}$ & $\begin{array}{l}1518.79+2459.6 \\
1531.18+2415.8 \\
1537.80+2635.1\end{array}$ & $\begin{array}{l}0.7 \\
0.8 \\
0.7\end{array}$ & & & & $\begin{array}{l}\mathrm{Sm} \\
\mathrm{dI} / \mathrm{Im} \\
\mathrm{dI}\end{array}$ & $\begin{array}{l}-642 \rightarrow 22471 \\
-642 \rightarrow 15275 \\
-642 \rightarrow 15275\end{array}$ \\
\hline F508-V1 & $1302.13+2702.7$ & 0.7 & & & & $\mathrm{dI} / \mathrm{Im}$ & \\
\hline F511-V1 & $1430.56+2259.1$ & 0.8 & 6294 & 1.13 & 131 & $\mathrm{Sm}:$ & \\
\hline F512-V1 & $1443.31+2553.9$ & 0.6 & & & & $\mathrm{Sm}$ & \\
\hline F513-V1 & $1508.61+2613.0$ & 0.7 & 10015 & 0.72 & 197 & S/Malin-like & \\
\hline F515-V1 & $1559.80+2638.6$ & 0.7 & & & & ring & $-642 \rightarrow 15275$ \\
\hline F520-V1 & $1744.83+2449.6$ & 0.9 & & & & $\mathrm{Sc} / \mathrm{Irr}$ & \\
\hline F532-V1 & $2200.49+2552.0$ & 0.8 & & & & ring & $-642 \rightarrow 32039$ \\
\hline F533-V1 & $2233.80+2327.2$ & 1.0 & & & & $\mathrm{Sd}$ & $-1908 \rightarrow 13220$ \\
\hline F535-V1 & $2312.71+2529.6$ & 0.8 & & & & $\mathrm{Im} / \mathrm{Sm}$ & \\
\hline $\begin{array}{l}\text { F539-V1 } \\
\text { F539-V2 }\end{array}$ & $\begin{array}{l}0008.87+2109.6 \\
0013.46+1815.7\end{array}$ & $\begin{array}{l}0.8 \\
0.5\end{array}$ & 7931 & 2.22 & 162 & $\begin{array}{l}\mathrm{Sm} \\
\mathrm{Sb}\end{array}$ & \\
\hline F542-V1 & $0116.76+1933.0$ & 0.6 & & & & Sb: & \\
\hline F544-V1 & $0209.71+1815.1$ & 0.8 & 5313 & 1.19 & 109 & $\mathrm{Sm}: /$ near dwarf & \\
\hline $\begin{array}{l}\text { F549-V1 } \\
\text { F549-V2 }\end{array}$ & $\begin{array}{l}0344.04+2111.0 \\
0401.60+2114.6\end{array}$ & $\begin{array}{l}0.7 \\
0.6\end{array}$ & $\begin{array}{l}5777 \\
6405\end{array}$ & $\begin{array}{l}1.70 \\
1.54\end{array}$ & $\begin{array}{r}149 \\
70\end{array}$ & $\begin{array}{l}\mathrm{Sm} \\
\mathrm{Im}\end{array}$ & \\
\hline $\begin{array}{l}\text { F562-V1 } \\
\text { F562-V2 }\end{array}$ & $\begin{array}{l}0833.93+1950.7 \\
0829.81+1941.0\end{array}$ & $\begin{array}{l}0.6 \\
0.9\end{array}$ & $\begin{array}{l}4647 \\
6330\end{array}$ & $\begin{array}{l}2.06 \\
2.32\end{array}$ & $\begin{array}{r}109 \\
78\end{array}$ & $\begin{array}{l}\operatorname{Irr} / \mathrm{Sm} \\
\mathrm{S}:\end{array}$ & \\
\hline $\begin{array}{l}\text { F563-V1 } \\
\text { F563-V2 }\end{array}$ & $\begin{array}{l}0843.80+1904.4 \\
0850.25+1837.6\end{array}$ & $\begin{array}{l}0.6 \\
0.9\end{array}$ & $\begin{array}{l}3936 \\
4311\end{array}$ & $\begin{array}{l}0.80 \\
2.61\end{array}$ & $\begin{array}{r}78 \\
118\end{array}$ & $\begin{array}{l}\mathrm{dI} \\
\mathrm{Irr}\end{array}$ & \\
\hline $\begin{array}{l}\text { F564-V1 } \\
\text { F564-V2 } \\
\text { F564-V3 }\end{array}$ & $\begin{array}{l}0905.32+2212.3 \\
0902.48+2149.5 \\
0900.05+2016.4\end{array}$ & $\begin{array}{l}0.8 \\
0.8 \\
0.7\end{array}$ & 481 & 1.52 & 48 & $\begin{array}{l}\text { E/interacting pair } \\
\mathrm{dI} \\
\mathrm{dI} / \mathrm{dE}\end{array}$ & $2961 \rightarrow 23500$ \\
\hline F565-V1 & $0916.64+2148.9$ & 0.7 & 491 & 0.26 & 27 & $\mathrm{dI}$ & \\
\hline F565-V2 & $0934.79+2159.8$ & 0.8 & 3681 & 0.97 & 97 & Im & \\
\hline
\end{tabular}


TABLE 2. (continued)

\begin{tabular}{|c|c|c|c|c|c|c|c|}
\hline Object & RA (1950) Dec & $\mathrm{D}$ & $V_{H I}$ & $S_{\nu}$ & $W_{50}$ & Notes & Search Range \\
\hline $\begin{array}{l}\text { F565-V3 } \\
\text { F565-V4 }\end{array}$ & $\begin{array}{l}0929.98+2141.0 \\
0927.39+2012.7\end{array}$ & $\begin{array}{l}0.8 \\
0.7\end{array}$ & 543 & 1.85 & 129 & $\begin{array}{l}\mathrm{dE} \\
\mathrm{dE} / \mathrm{dI}\end{array}$ & $1013 \rightarrow 9111$ \\
\hline F568-V1 & $1042.33+2219.3$ & 0.7 & 5769 & 2.13 & 161 & S/Malin-like & \\
\hline F570-V1 & $1120.38+1745.5$ & 0.8 & 1382 & 0.39 & 71 & dI & \\
\hline F571-V1 & $1123.69+1906.4$ & 0.9 & 5704 & 0.85 & 65 & $\mathrm{Sd} / \mathrm{Sm}$ & \\
\hline F577-V1 & $1347.77+1831.0$ & 0.8 & 7776 & 1.42 & 58 & Sd: & \\
\hline F579-V1 & $1430.53+2259.0$ & 0.8 & & & & Sc & \\
\hline F581-V1 & $1504.33+2040.9$ & 0.8 & 6233 & 1.20 & 40 & Sd: & \\
\hline F582-V1 & $1525.44+2140.2$ & 0.5 & 11695 & 1.23 & 178 & ring & \\
\hline $\begin{array}{l}\text { F583-V1 } \\
\text { F583-V2 }\end{array}$ & $\begin{array}{r}.1548 .12+2054.8 \\
1546.46+2050.2\end{array}$ & $\begin{array}{l}0.6 \\
0.7\end{array}$ & $\begin{array}{l}10926 \\
11011\end{array}$ & $\begin{array}{l}1.12 \\
1.14\end{array}$ & $\begin{array}{l}212 \\
146\end{array}$ & $\begin{array}{l}\mathrm{Sc} \\
\mathrm{Sb}\end{array}$ & \\
\hline F585-V1 & $1623.80+2046.6$ & 0.4 & & & & dI & \\
\hline F597-V1 & $2041.90+2154.7$ & 0.8 & & & & S/ring & \\
\hline $\begin{array}{l}\text { F602-V1 } \\
\text { F602-V2 } \\
\text { F602-V3 }\end{array}$ & $\begin{array}{l}2215.00+1944.4 \\
2228.91+2110.4 \\
2229.79+1822.6\end{array}$ & $\begin{array}{l}0.6 \\
0.8 \\
0.7\end{array}$ & $\begin{array}{l}7241 \\
7243\end{array}$ & $\begin{array}{l}1.42 \\
2.93\end{array}$ & $\begin{array}{r}66 \\
187\end{array}$ & $\begin{array}{l}\mathrm{Sm} \\
\mathrm{Sm} \\
\mathrm{Im} / \mathrm{Sm}\end{array}$ & $-642 \rightarrow 15275$ \\
\hline $\begin{array}{l}\text { F608-V1 } \\
\text { F608-V2 }\end{array}$ & $\begin{array}{l}0010.20+1414.8 \\
0020.51+1451.8\end{array}$ & $\begin{array}{l}0.6 \\
0.8\end{array}$ & $\begin{array}{l}1828 \\
5554\end{array}$ & $\begin{array}{l}2.67 \\
0.62\end{array}$ & $\begin{array}{r}105 \\
85\end{array}$ & $\begin{array}{l}\text { Sd } \\
\text { Sd }\end{array}$ & \\
\hline $\begin{array}{l}\text { F611-V1 } \\
\text { F611-V2 } \\
\text { F611-V3 }\end{array}$ & $\begin{array}{l}0113.63+1600.3 \\
0122.59+1347.8 \\
0125.28+1414.6\end{array}$ & $\begin{array}{l}0.7 \\
0.8 \\
0.5\end{array}$ & & & & $\begin{array}{l}\text { S/Malin-like } \\
\text { Im } \\
\text { SBm }\end{array}$ & $-642 \rightarrow 32039$ \\
\hline $\begin{array}{l}\text { F612-V1 } \\
\text { F612-V2 } \\
\text { F612-V3 }\end{array}$ & $\begin{array}{l}0134.76+1425.2 \\
0135.62+1509.1 \\
0138.65+1714.7\end{array}$ & $\begin{array}{l}0.8 \\
0.8 \\
0.6\end{array}$ & $\begin{array}{r}2589 \\
21550 \\
5005\end{array}$ & $\begin{array}{l}1.60 \\
0.97 \\
0.81\end{array}$ & $\begin{array}{r}50 \\
385 \\
62\end{array}$ & $\begin{array}{l}\text { Irr } \\
\text { S/Malin-like } \\
\text { dI/Im }\end{array}$ & \\
\hline $\begin{array}{l}\text { F613-V4 } \\
\text { F613-V5 }\end{array}$ & $\begin{array}{l}0147.44+1608.6 \\
0151.26+1313.1\end{array}$ & $\begin{array}{l}0.7 \\
0.9\end{array}$ & $\begin{array}{l}5023 \\
6200\end{array}$ & $\begin{array}{l}0.92 \\
1.50\end{array}$ & $\begin{array}{l}81 \\
29\end{array}$ & $\begin{array}{l}\mathrm{Im} / \mathrm{dI} \\
\mathrm{Sm}\end{array}$ & \\
\hline $\begin{array}{l}\text { F614-V1 } \\
\text { F614-V2 } \\
\text { F614-V3 }\end{array}$ & $\begin{array}{l}0218.17+1523.8 \\
0219.01+1614.8 \\
0219.93+1700.0\end{array}$ & $\begin{array}{l}0.8 \\
0.7 \\
0.5\end{array}$ & $\begin{array}{l}3953 \\
4047\end{array}$ & $\begin{array}{l}1.31 \\
1.43\end{array}$ & $\begin{array}{l}30 \\
63\end{array}$ & $\begin{array}{l}\text { Sd } \\
\text { dI } \\
\text { dI }\end{array}$ & $-642 \rightarrow 15275$ \\
\hline $\begin{array}{l}\text { F620-V1 } \\
\text { F620-V2 } \\
\text { F620-V3 }\end{array}$ & $\begin{array}{l}0416.20+1442.5 \\
0420.31+1612.0 \\
0429.10+1441.0\end{array}$ & $\begin{array}{l}0.7 \\
0.7 \\
0.5\end{array}$ & & & & $\begin{array}{l}\text { S/smooth } \\
\text { Sm } \\
\text { dI }\end{array}$ & \\
\hline $\begin{array}{l}\text { F634-V1 } \\
\text { F634-V2 }\end{array}$ & $\begin{array}{l}0900.65+1550.5 \\
0908.36+1319.5\end{array}$ & $\begin{array}{l}0.7 \\
0.9\end{array}$ & 8899 & 2.51 & 126 & $\begin{array}{l}\text { Im } \\
\text { S/Malin-like }\end{array}$ & $-642 \rightarrow 23500$ \\
\hline F635-V1 & $0914.15+1456.3$ & 0.7 & & & & $\operatorname{Im}$ & \\
\hline $\begin{array}{l}\text { F638-V1 } \\
\text { F638-V2 }\end{array}$ & $\begin{array}{l}1012.11+1439.7 \\
1015.89+1506.3\end{array}$ & $\begin{array}{l}0.5 \\
0.7\end{array}$ & & & & $\begin{array}{l}\text { Sm/Im } \\
\text { S/Malin-like }\end{array}$ & $-642 \rightarrow 23500$ \\
\hline $\begin{array}{l}\text { F640-V1 } \\
\text { F640-V2 }\end{array}$ & $\begin{array}{l}1053.62+1216.6 \\
1054.99+1414.5\end{array}$ & $\begin{array}{l}0.7 \\
0.8\end{array}$ & $\begin{array}{r}990 \\
1239\end{array}$ & $\begin{array}{l}1.44 \\
2.31\end{array}$ & $\begin{array}{l}32 \\
93\end{array}$ & $\begin{array}{l}\operatorname{Im} / \mathrm{Sm} \\
\mathrm{Irr}\end{array}$ & \\
\hline $\begin{array}{l}\text { F640-V3 } \\
\text { F640-V4 }\end{array}$ & $\begin{array}{l}1058.23+1409.1 \\
1109.89+1634.0\end{array}$ & $\begin{array}{l}0.9 \\
0.7\end{array}$ & & & & $\begin{array}{l}\mathrm{dE}: / \text { near N3489 } \\
\mathrm{dE}\end{array}$ & $-642 \rightarrow 15275$ \\
\hline
\end{tabular}




\begin{tabular}{|c|c|c|c|c|c|c|c|}
\hline Object & RA (1950) Dec & $\mathrm{D}$ & $V_{H I}$ & $S_{\nu}$ & $W_{50}$ & Notes & Search Range \\
\hline F648-V1 & $1338.22+1720.6$ & 0.8 & & & & $\mathrm{Sm}$ & \\
\hline F648-V2 & $1340.27+1552.2$ & 0.9 & & & & S/Malin-like & \\
\hline F650-V1 & $1412.97+1428.9$ & 0.7 & 5249 & 1.76 & 37 & $\mathrm{Sd} / \mathrm{Im}$ & \\
\hline F650-V2 & $1427.73+1552.9$ & 0.8 & 8313 & 1.44 & 191 & $\mathrm{Sm} / \mathrm{Im}$ & \\
\hline F650-V3 & $1429.14+1725.5$ & 0.6 & 8806 & 1.09 & 140 & $\operatorname{Im}$ & \\
\hline F651-V1 & $1442.05+1243.0$ & 0.6 & 8895 & 1.01 & 63 & $\operatorname{Im}$ & \\
\hline F672-V1 & $2147.18+1538.4$ & 0.5 & & & & $\mathrm{Sc} / \mathrm{Sm}$ & $-642 \rightarrow 15275$ \\
\hline F672-V2 & $2147.25+1537.2$ & 0.5 & & & & Im: & $-642 \rightarrow 15275$ \\
\hline F674-V1 & $2209.09+1529.1$ & 0.6 & 6047 & 0.65 & 111 & $\mathrm{Im} / \mathrm{dI}$ & \\
\hline F675-V1 & $2238.88+1627.1$ & 0.7 & 3208 & 0.25 & 30 & Irr & \\
\hline F675-V2 & $2245.15+1458.8$ & 0.6 & & & & $\mathrm{Sm}$ & $-642 \rightarrow 7365$ \\
\hline F677-V1 & $2310.32+1347.6$ & 0.7 & 4705 & 3.48 & 108 & $\operatorname{Im}$ & \\
\hline F677-V2 & $2309.72+1718.6$ & 0.6 & 4969 & 0.52 & 36 & $\mathrm{Im} / \mathrm{dI}$ & \\
\hline F677-V3 & $2321.67+1536.6$ & 0.5 & & & & $\mathrm{dI} /$ double? & $-642 \rightarrow 15275$ \\
\hline F677-V4 & $2322.77+1226.5$ & 0.7 & 3664 & 1.42 & 29 & $\mathrm{dI}$ & \\
\hline F677-V5 & $2323.71+1448.1$ & 0.5 & & & & $\mathrm{dI} / \mathrm{Im}$ & $-642 \rightarrow 7365$ \\
\hline F677-V6 & $2325.07+1327.3$ & 0.8 & 3882 & 1.25 & 113 & $\mathrm{Sm} / \mathrm{Im}$ & \\
\hline $\mathrm{F} 677-\mathrm{V} 7$ & $2325.96+1656.1$ & 0.5 & & & & $\mathrm{dI}$ & $-642 \rightarrow 7365$ \\
\hline F682-V1 & $0054.07+1004.4$ & 0.5 & 10410 & 1.27 & 154 & $\mathrm{Sc}$ & \\
\hline F682-V2 & $0059.22+1000.3$ & 0.5 & 11428 & 0.70 & 115 & $\mathrm{Sm}$ & \\
\hline F683-V1 & $0117.37+0836.1$ & 0.5 & & & & $\mathrm{Sm} / \mathrm{N} 524$ cluster & \\
\hline F683-V2 & $0119.47+0909.6$ & 0.8 & 5680 & 0.29 & 91 & $\mathrm{Im} / \mathrm{N} 524$ cluster & \\
\hline F683-V3 & $0119.78+0911.4$ & 0.5 & & & & $\mathrm{Im} / \mathrm{real}$ ?/N524 cluster & $-642 \rightarrow 15275$ \\
\hline F683-V4 & $0120.04+0853.3$ & 0.5 & & & & $\mathrm{Sm} / \mathrm{Im} / \mathrm{N} 524$ cluster & \\
\hline F683-V5 & $0120.07+0909.4$ & 0.4 & & & & $\mathrm{Sm} / \mathrm{Im} / \mathrm{N} 524$ cluster & \\
\hline F683-V6 & $0120.44+0904.9$ & 0.7 & & & & $\mathrm{Sd} / \mathrm{N} 524$ cluster & \\
\hline F683-V7 & $0122.47+0944.8$ & 0.5 & & & & $\mathrm{Sm} / \mathrm{N} 524$ cluster & $-642 \rightarrow 7365$ \\
\hline F683-V8 & $0123.15+0919.4$ & 0.6 & & & & $\mathrm{Sb} / \mathrm{N} 524$ cluster & \\
\hline F683-V9 & $0124.89+0922.7$ & 0.5 & & & & $\mathrm{Sm} / \mathrm{N} 524$ cluster & $-642 \rightarrow 7365$ \\
\hline F683-V10 & $0127.92+1114.0$ & 0.5 & 974 & 1.17 & 50 & $\mathrm{dI} / \mathrm{N} 524$ cluster & \\
\hline F687-V1 & $0237.96+0841.9$ & 0.7 & 5746 & 0.48 & 42 & $\mathrm{SBm}$ & $\cdot$ \\
\hline F687-V2 & $0242.59+0920.5$ & 0.6 & 3390 & 0.68 & 62 & $\mathrm{Sm} / \mathrm{Im}$ & \\
\hline F702-V1 & $0735.22+1018.4$ & 0.8 & & & & S: (ZWG058.017) & \\
\hline F704-V1 & $0822.16+0923.2$ & 0.7 & 6016 & 2.19 & 121 & S/Malin-like & \\
\hline F721-V1 & $1350.77+0955.6$ & 0.7 & 11346 & 0.97 & 80 & ring & \\
\hline F721-V2 & $1353.48+0915.5$ & 0.8 & 1222 & 0.23 & 52 & $\mathrm{dI}$ & \\
\hline F721-V3 & $1358.07+0914.1$ & 0.5 & 4148 & 0.83 & 102 & $\operatorname{Im}$ & \\
\hline F721-V4 & $1400.28+1013.9$ & 0.6 & 5811 & 0.80 & 94 & S/Malin-like & \\
\hline F723-V1 & $1430.08+1146.3$ & 0.9 & & & & $\mathrm{Im} / \mathrm{SBm}:$ & \\
\hline F723-V2 & $1434.27+1147.7$ & 0.8 & & & & $\mathrm{Sb}$ (ZWG075.090) & \\
\hline F723-V3 & $1442.07+1237.2$ & 0.7 & & & & $\operatorname{Im}$ & \\
\hline F723-V4 & $1448.20+0834.7$ & 0.7 & & & & $\mathrm{SBc}$ & \\
\hline F725-V1 & $1520.68+1156.4$ & 0.8 & 7278 & 1.99 & 142 & $\mathrm{Sm}$ & \\
\hline F726-V1 & $1532.70+0742.5$ & 0.7 & & & & ring & \\
\hline
\end{tabular}


TABLE 2. (continued)

\begin{tabular}{llllllll}
\hline Object & RA (1950) Dec & $\mathrm{D}$ & $V_{H I}$ & $S_{\nu}$ & $W_{50}$ & Notes & Search Range \\
\hline F726-V2 & $1537.39+0836.5$ & 0.7 & 4061 & 1.53 & 140 & Sm & \\
F726-V3 & $1539.53+1036.6$ & 0.8 & 5625 & 1.48 & 63 & S/Malin-like & \\
F727-V1 & $1549.26+0901.2$ & 0.6 & & & & Sc & \\
F727-V2 & $1602.16+1105.4$ & 0.9 & & & & Sb & \\
F727-V3 & $1602.84+1110.5$ & 0.8 & & & & Sm & \\
F727-V4 & $1610.53+1115.0$ & 0.7 & & & & S/Malin-like & \\
F728-V1 & $1610.63+1025.5$ & 0.7 & 9672 & 0.67 & 46 & S/Malin-like & \\
F728-V3 & $1629.09+1118.9$ & 0.6 & & & & ring & \\
F730-V1 & $1701.58+1014.9$ & 0.8 & & & & Sm & \\
F745-V1 & $2154.83+0805.4$ & 0.6 & 3484 & 3.53 & 37 & Irr & \\
F745-V2 & $2155.34+1046.2$ & 0.4 & & & & Sc(r) & $-642 \rightarrow 7365$ \\
F746-V1 & $2214.75+0938.7$ & 0.6 & & & & S(r) & $-642 \rightarrow 7365$ \\
F746-V2 & $2231.08+1115.4$ & 0.7 & & & & Irr & $-642 \rightarrow 7365$ \\
F750-V1 & $2351.77+1039.9$ & 0.8 & 944 & 0.66 & 32 & Irr & \\
\hline \hline
\end{tabular}

attempt to characterize a category exemplified by Malin-1 (Bothun et al. 1987). Several examples of these large diameter, high redshift objects now exist (e.g., F568-6, Bothun et al. 1990), but it is unclear whether or not they can be identified solely by their morphology. The class can basically be summarized as a very LSB, fragmented spiral. These objects have distinct bulges (sometimes the bulge was identified in the Zwicky CGCG catalog as an elliptical) embedded in a very LSB disk. The outer regions of these disk often have low luminosity knots which $\mathrm{H} \alpha$ images show are large $\mathrm{H}$ II regions. If the spiral pattern were stronger or the bulges smaller then these types of galaxies would fit into the late type spiral sequence. However, the bulges are prominent, making them inconsistent with an $\mathrm{Sm}$ classification. The classification as $\mathbf{S} /$ Malin-like is noted in the catalog if there is a similarity to the above description using F568-6 from Fig. 2 of Paper I as the prototype. Evidence that this classification corresponds to a real galaxy type comes from the fact that $50 \%$ of the objects noted as Malin-like are indeed high in velocity and large in absolute size (see Sec. 4.2).

\section{DISCUSSION}

\subsection{Morphology of LSB Galaxies}

Not surprisingly, the LSB catalog is dominated by late type galaxies. This is due primarily to the definition and the selection process: since ellipticals have steep profiles, few should be missed in a diameter limited catalog. Figure 1 displays a histogram of the various morphological types in the catalog. Only a handful of ellipticals are in the LSB catalog for the reasons stated above and the few that are present are surrounded by faint halos (cD-like or shell ellipticals). A few S0's with LSB disks are present in the catalog, but not in the same proportion as other LSB disk systems. It may well be that the phenomenon that is responsible for a LSB disk in a late type galaxy does not operate in S0's. A population study of SO's over a range of disk surface brightnesses would be illuminating since LSB S0's are so rare.

Approximately $14 \%$ of the primary catalog is early type spirals. However, during the early phase of the catalog there was a conflict in the morphological types of LSB galaxies for early type spirals ( $\mathrm{Sa}$ to $\mathrm{Sb}$ ) and the traditional Hubble definition. Many of the objects with Sa type arms had small bulges more typical of the Sc type spirals. Reexamination of these objects showed that many of the bulges were, in fact, stellar-like, and that the true bulge was hidden below the brightness of the nucleus. Followup spectroscopy will probably show that these nuclei are low intensity AGN in nature, as are the few Malin-like spirals studied (Bothun et al. 1990). Confirmation for a larger sample is required before concluding that low level AGN activity is fairly common in a sample of LSB disk systems for reasons unknown at this time. Once this problem was identified, and the early type spirals recovered from misclassification, the proportion of early type spirals in the sample is surprisingly high. It has often been naively assumed that only dwarfs and small spirals are low in surface brightness, reflecting past histories of weak star formation associated with low-mass density. However, many of these early type spirals have similar characteristics to their HSB cousins, including size and arm design. They simply represent the same morphological style as their counterparts in the Hubble Atlas differing in having much lower average and central surface brightnesses. Further proof of the close relationship between the HSB and LSB spirals is found from $\mathrm{H}$ I observations in Sec. 4.2, but it is important to note that the realm of LSB galaxies is well represented in all spiral types and not just the late types.

As can be seen in Fig. 1, a majority of LSB galaxies fall in the Sc, Sm, and Im classes. Compared to the UGC, the mean type found in the LSB search is considerably later. This suggests that searches to a deeper limiting isophote is primarily a process of recovering the far end of the Hubble sequence. These galaxies, however, can be either dwarfs or giant disk systems, the extreme opposite ends of the range of galaxy scale length. This is an important distinction since some LSB surveys have been optimized to recover dwarf galaxies and to explore the faint end of the luminosity function (e.g., Bothun et al. 1991). In point of fact, both ends of the scale 


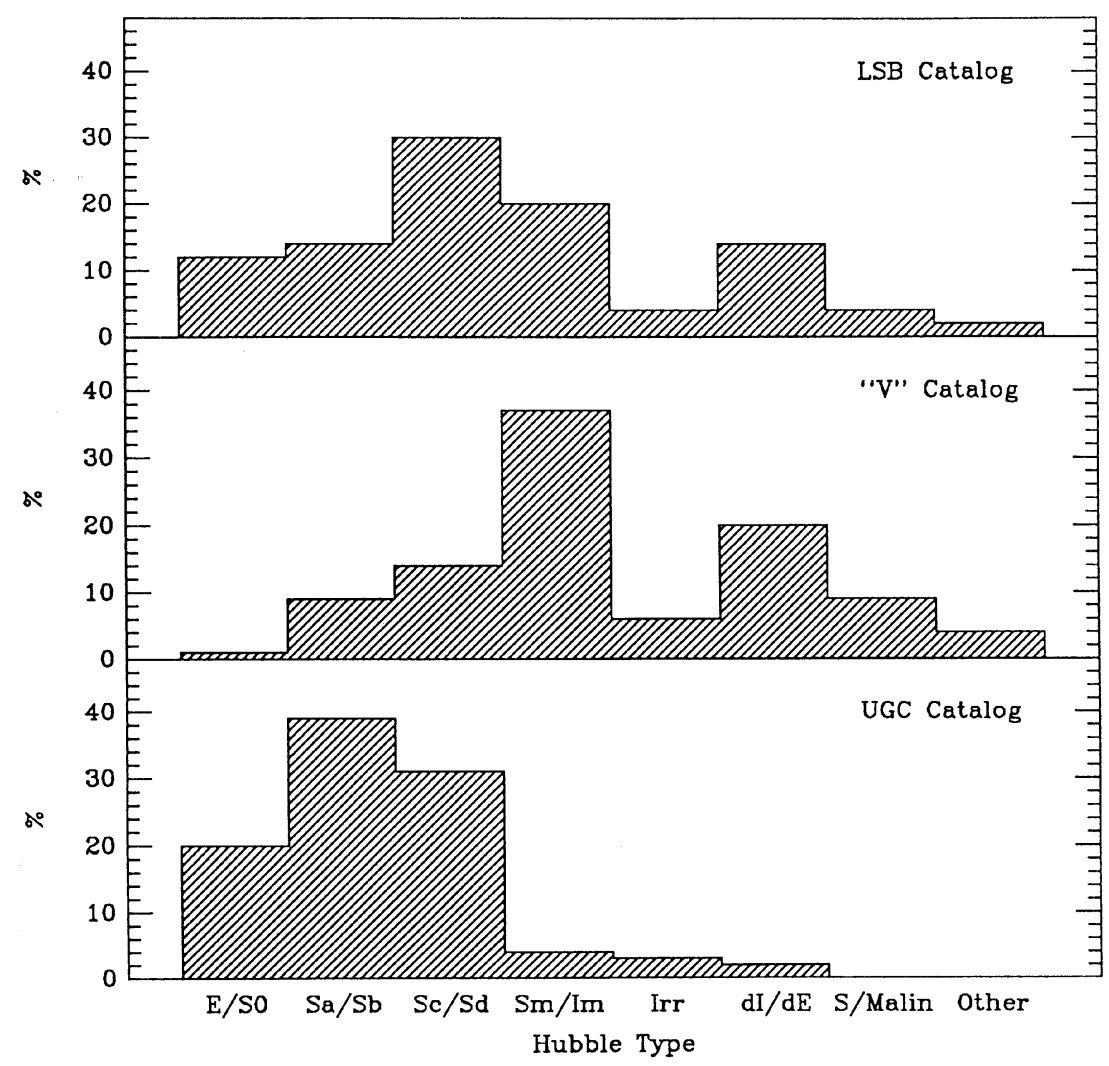

FIG. 1. Morphological breakdown of the LSB catalog. As is expected in a LSB sample the catalog is dominated by late type galaxies as compared to the UGC catalog. See text for description of the Hubble classes used.

length distribution of galaxies are poorly understood because of surface brightness bias (see Bothun 1990).

Dwarf galaxies of the $\mathrm{dI}$ and $\mathrm{dE}$ class constitute about $15 \%$ of the catalog. Classification of these objects is extremely difficult as they approach the detection limit such that there is probably a severe overlap in the $\mathrm{dE} / \mathrm{dI} / \mathrm{Im}$ categories. Uncertainty is represented by double classification (e.g., $\mathrm{dE} / \mathrm{dI}$ or $\mathrm{Im} / \mathrm{dI}$ ) where the first type is the most probable in the opinion of the classifier. Some of these ambiguities are resolved if the galaxy is detected in $\mathrm{H} \mathrm{I}$; thus a dI classification is more likely than $\mathrm{dE}$ if gas is detected, regardless of the morphological considerations. Figure 2 displays the distribution of dwarfs in the sky. As can be seen, all but two dE's (F415-1 and F620-3) are located within $15^{\circ}$ of the Virgo cluster which is confirmation of the strong morphological segregation among dwarfs observed by Binggeli et al. (1991).

\subsection{H I Redshifts and Masses}

By their nature, LSB galaxies require a prohibitive amount of telescope time to obtain optical spectroscopy.
Thus redshifts must come from detections at $21 \mathrm{~cm}$. Fortunately, most LSB disk galaxies are rich in $\mathrm{H}$ I. For this reason, the initial phase of the PSS-II search for LSB galaxies is being conducted in the declination range accessible to the Arecibo radio telescope. The results of our $\mathrm{H}$ i survey to date are summarized in Tables 1 and 2. For a more detailed description of the reduction procedures and the errors the reader is referred to Schneider et al. (1990).

The redshift distribution for the primary and " $\mathrm{V}$ " catalog is shown in Fig. 3. Also shown in the redshift distribution of UGC galaxies as taken from the $\mathrm{H}$ I catalog of Huchtmeier \& Richter (1983) restricted to galaxies with angular sizes less than 4 arcmin. The LSB distribution can be divided into three classes: dwarfs between - 500 and $3000 \mathrm{~km} \mathrm{~s}^{-1}$, LSB disk galaxies, and large-scale length disk galaxies (Malin objects in the terminology of Impey \& Bothun 1989). The later category is reserved for all galaxies with velocities greater than $10000 \mathrm{~km} \mathrm{~s}^{-1}$. A majority of these have a morphological appearance similar to Malin-1 and F568-6; however, there are exceptions. The redshift distribution is similar to the distribution from the higher surface brightness galaxies of the UGC except for the low and high ends. At the low

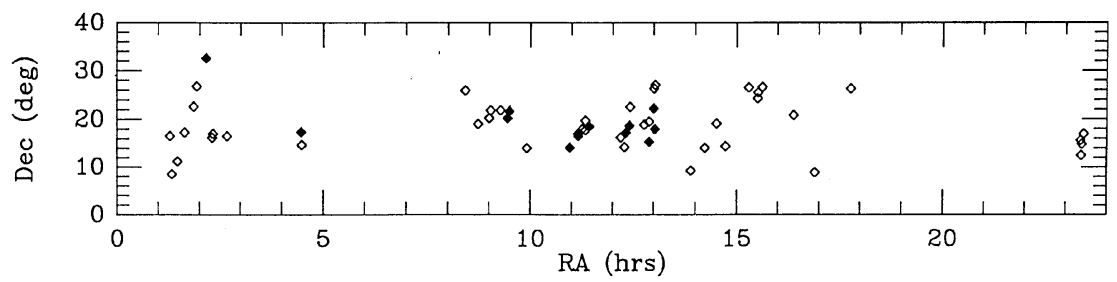

FIG. 2. Plot of the positions of dwarf galaxies from the LSB catalog. The solid symbols represent dwarf ellipticals (dE's) and the open symbols represent dwarf irregulars (dI's) as defined by morphology only (vs $\mathrm{H}$ I content). All but two dE's are within $15^{\circ}$ of the Virgo cluster. 


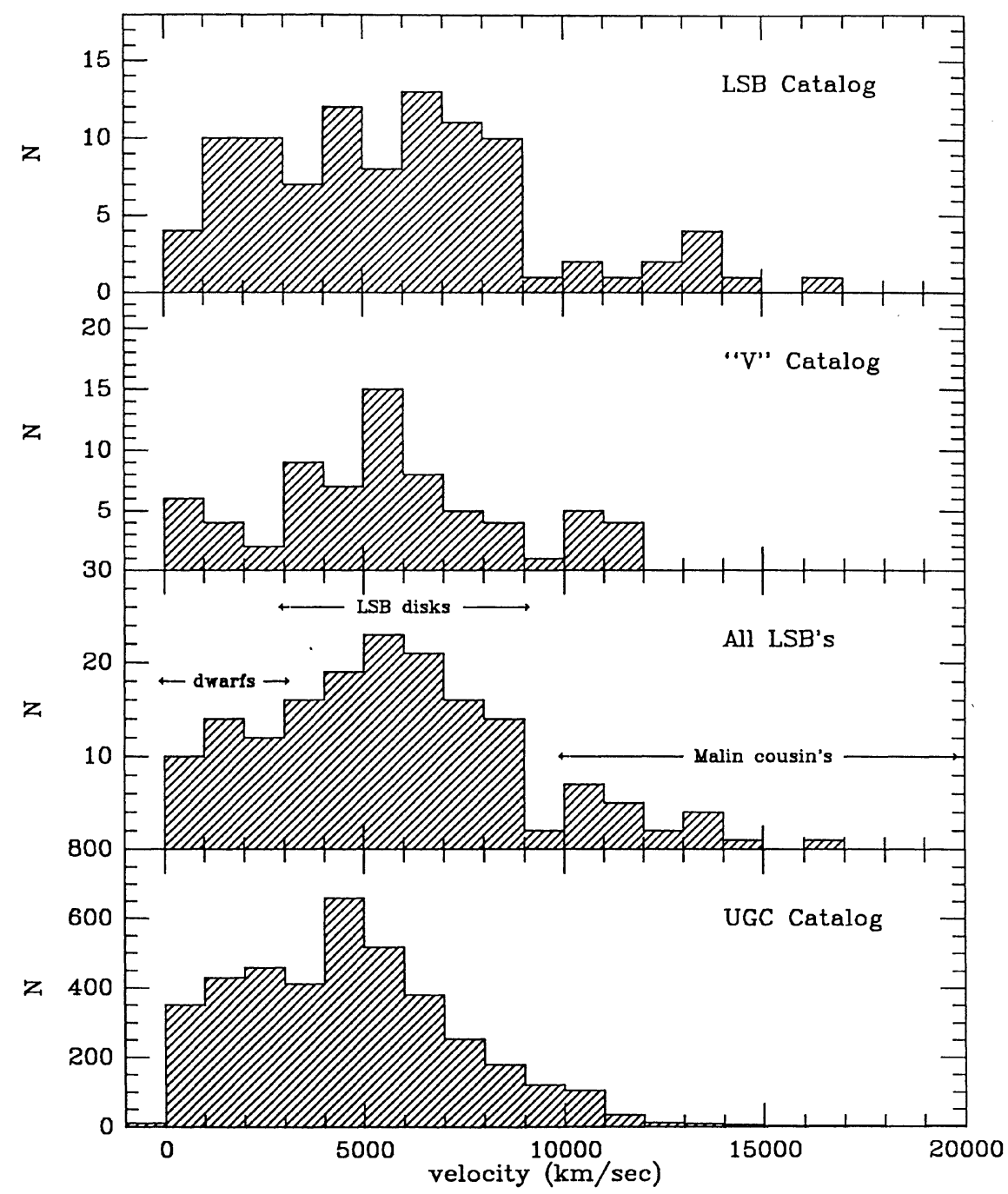

FIG. 3. Redshift histograms of the $\mathrm{H} \mathrm{I}$ detections in the LSB catalog. The top panel displays only the primary catalog, the second panel displays the " $\mathrm{V}$ " cata$\log$, and the third panel displays the sum. The distribution can be divided into three broad classes of galaxies; nearby dwarfs, LSB disks, and Malin objects. The redshift distribution of the UGC from the Huchtmeier and Richter cata$\log$ is shown for comparison in the bottom panel. A majority of the objects between 2000 and $8000 \mathrm{~km} \mathrm{~s}^{-1}$ are spirals. The corresponding objects in the LSB catalog are called LSB disks.

velocity end, our survey finds proportionately more low-luminosity, small LSB disks. Most of these galaxies have physical diameters between 2 and $6 \mathrm{kpc}$. True dwarf galaxies are generally defined as objects with $M_{B}>-16$ and have diameters $<2 \mathrm{kpc}$ (e.g., Hodge et al. 1965). Thus, our visual scanning of the PSS-II does not preferentially select dwarf galaxies. These objects may better be selected using the techniques described in Impey et al. (1988). Without a sensitive all sky survey, one can not preclude the possibility that a significant fraction of the mass of the local Universe is bound up in high $\mathscr{M} / L$ extremely LSB dwarf galaxies (e.g., Bothun et al. 1991; Silk 1991). On the other hand, relative to the UGC, a higher percentage of Malin objects are discovered with deeper surface brightness surveys. These enigmatic objects are often in isolated regions of the Universe which suggests that galaxy size may be related to the local protogalaxy environment. Freed from interaction with other nearby condensations, these isolated gas clouds may well form large ( $D>100 \mathrm{kpc}$ ), low surface mass density structures, which subsequently evolve rather slowly over a Hubble time. The large cross section offered by these quiescent systems makes them excellent candidates for producing QSO absorption line systems, particularly damped Lyman $\alpha$ systems (e.g., Wolfe et al. 1986; Smith et al. 1989).

The redshift cone diagram for the catalog $\mathrm{H}$ I detections to date is shown in Fig. 4. Some of the features of the CfA redshift survey (de Lapparent et al. 1986) are present in the LSB catalog, such as the "Great Wall." By extension of the density-morphology relation (e.g., Dressler 1980; Postman \& Geller 1984) LSB galaxies (being predominately late type spirals) would be expected to be poor tracers of the mass on small scales as they avoid high density environs. This is confirmed in Fig. 4 which shows no sign of small-scale clustering. However, on scales $>5 \mathrm{~h}^{-1} \mathrm{Mpc}$, LSB galaxies appear to be excellent tracers of large-scale structure (e.g., Bothun et al. 1986, Thuan et al. 1991). Consistent with previous studies (but see Salzer et al. 1991 for a counter example) there is no indication of LSB galaxies "filling" the voids delineated by the CFA redshift survey of HSB (e.g., Zwicky) galaxies. A full treatment of this problem using nearestneighbor tests and cross-correlation functions will be presented at a later date when all the various LSB surveys are combined.

The distribution of $\mathrm{H}$ I mass is shown in Fig. 5 where all 


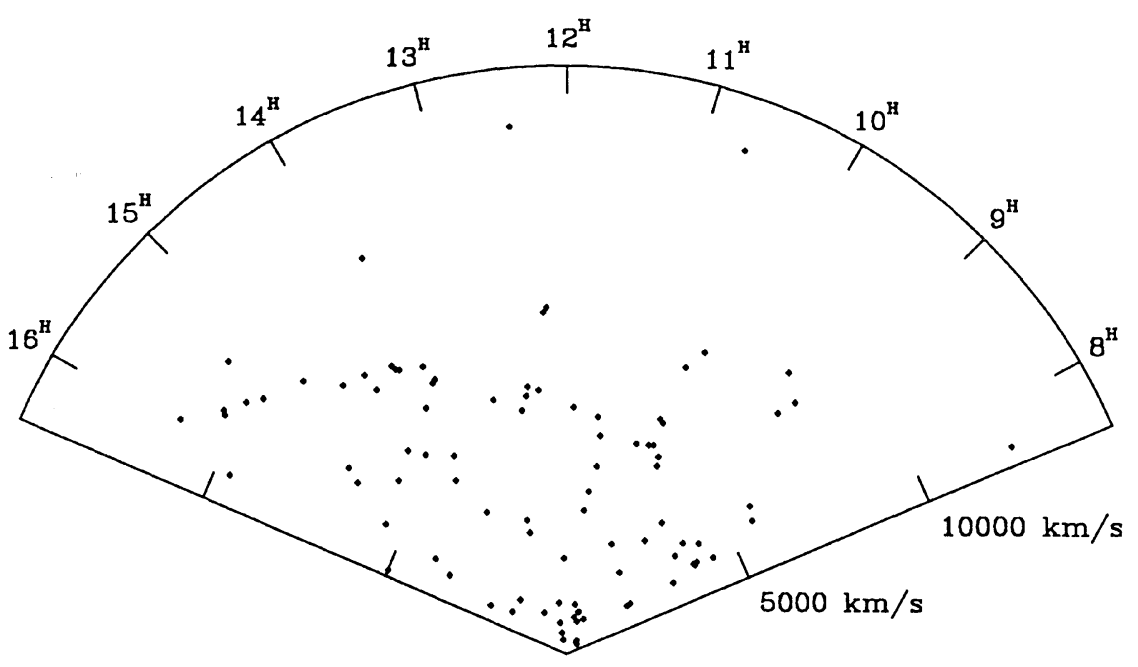

FIG. 4. Redshift cone diagrams for the LSB catalog. The "Great Wall" and other large-scale features are visible in the LSB sample. There is no indication that

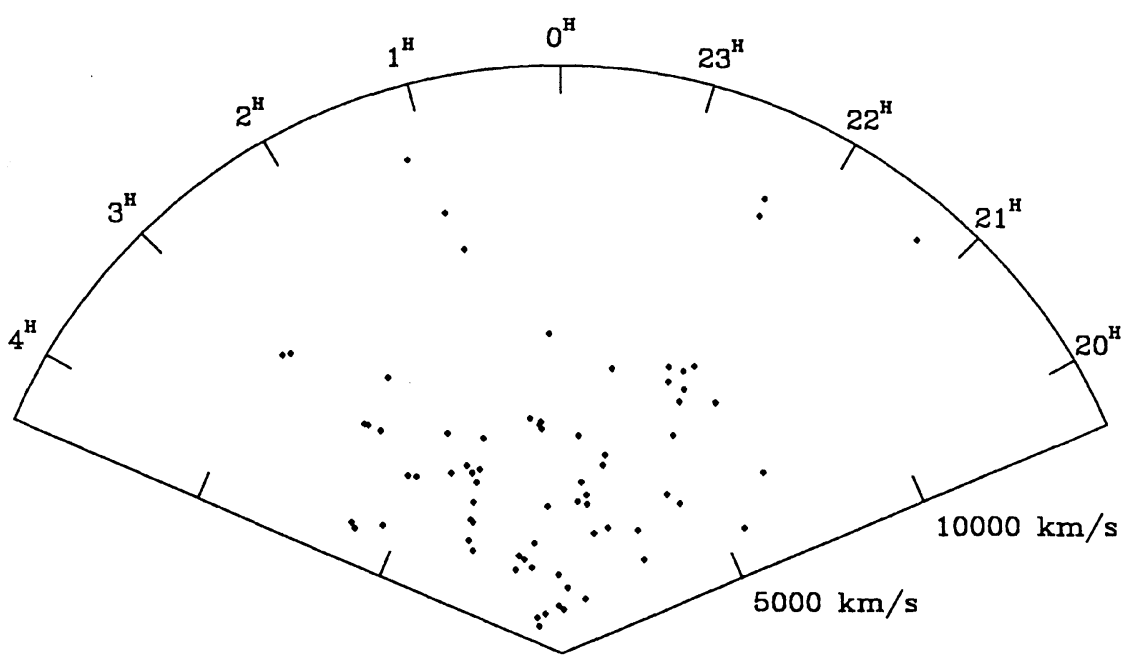
LSB galaxies fill voids or are, in general, distributed differently than HSB galaxies.

objects with velocities less than $1000 \mathrm{~km} \mathrm{~s}^{-1}$ or near Virgo infall cusps were ignored. Otherwise, the standard Galactic rotation value of $300 \mathrm{~km} \mathrm{~s}^{-1}$ was applied to a pure Hubble flow. The resulting distribution has a mean value of $1.3 \times 10^{9}$ $\mathscr{M}_{\odot}$ ranging from a minimum of $10^{7} \mathscr{M}_{\odot}$ to a maximum of $1.8 \times 10^{10} \mathscr{M}_{\odot}$. The $\mathrm{H}$ I masses from the UGC catalog as tabulated by Huchtmeier and Richter are shown for comparison in the bottom panel of Fig. 5. Aside from a slightly smaller mean, the two distributions are identical. At the very least, the $\mathrm{H}$ I masses of an LSB sample are not significantly lower than "normal" galaxies. This similarity of global $\mathrm{H}_{\mathrm{I}}$ properties coupled with the obvious disparity in global optical properties reinforces the idea that disk galaxy evolution is strongly tied to local disk conditions (see also van der Hulst et al. 1987).

The distribution of $\mathrm{H} \mathrm{I}$ widths is shown in Fig. 6 as $W_{50}$ the width of the profile at $50 \%$ peak value. The bottom panel displays the distribution for those profiles with clear doublehorned shape, indicating rotating disks. The upper panel displays the distribution for those profiles which were single horned or Gaussian in shape. No correction has been made for inclination since the axial ratios of these LSB galaxies is difficult to determine. In fact, there is a strong bias in the visual selection of LSB galaxies to preferentially find face-on objects. Thus, Fig. 6 displays the lower bounds of rotation velocity for the sample as a whole and again shows that this catalog is not a selection of objects with dwarf masses but rather covers a full range of galaxy masses. A breakdown by morphological type is shown in Fig. 7. The high-velocity objects are shown in the bottom panel and have a similar distribution to the early type spirals. Note that no inclination corrections have been made and that, if there is a selection effect to only identify Malin objects when face-on, then their distribution would only increase relative to the early type spirals.

\subsection{Optical Imaging and Star Formation}

The optical images of 41 of the members of the LSB cata$\log$ are shown in Fig. 8. The first ten panels display a broadband red image next to an $\mathrm{H} \alpha$ image. The eleventh and twelfth panel display a broadband and $\mathrm{H} \alpha$ picture of M101 

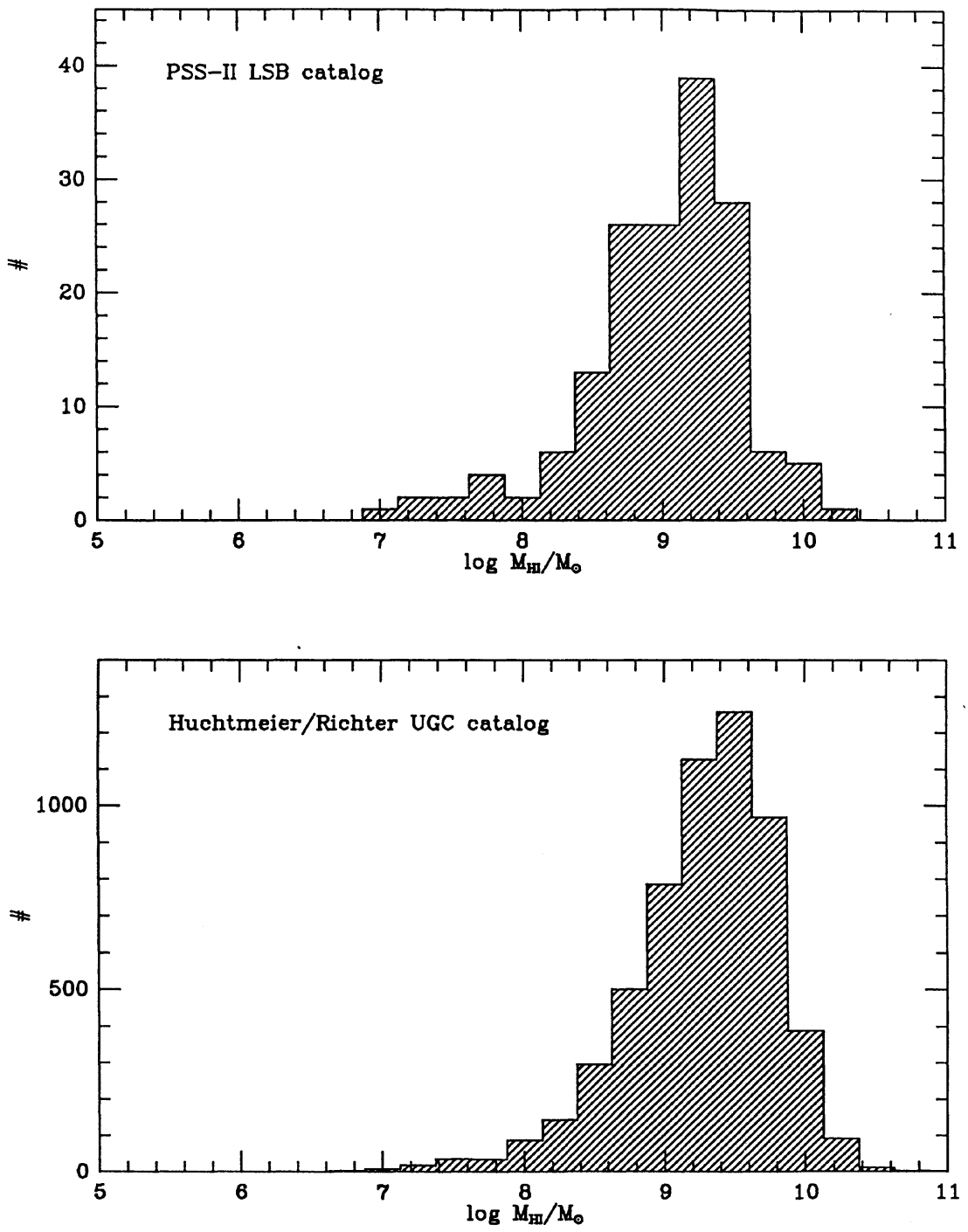

FIG. 5. H I mass histograms for the LSB catalog. $\mathrm{H}$ I masses for the objects in the LSB and UGC catalogs with velocities greater than $1000 \mathrm{~km} \mathrm{~s}^{-1}$. for comparison. The remaining 36 panels are broadband images of randomly selected members in order of their field numbers.

The broadband pictures reveal the most common characteristic of the catalog, namely, the objects are extremely low in contrast to the natural sky brightness. The greyscale software used to produce Fig. 8 overemphasizes the signal nearest sky and saturates regions only a few percent above sky. Thus, often the darkest regions in the LSB galaxies are near sky brightness themselves. With this higher contrast, most of the galaxies in Fig. 8 have features common in the Hubble sequence. Frequently there is a higher surface brightness galaxy embedded in a LSB envelope or disk (e.g., F530-3, F5333, F564-1, and F602-1). Another typical LSB galaxy type is shown by F568-1, a bulge embedded in a very LSB disk. Only the bulge (more of a central concentration then a true bulge) distinguishes this type from Sm's and Im's such as F614-1 or F533-1. When a spiral pattern is discernible, it is often smooth and grand design-like rather than fragmented. The differences between bulge and disk surface brightness reflect the differences in the first epoch of star formation (bulge) and later epochs (disk). A majority of the LSB galaxies have both low bulge surface brightness and disk surface brightness. For example, a typical spiral has a difference of 3-4 mag $\operatorname{arcsec}^{-2}$ between mean bulge and disk surface brightnesses; however, with the Malin objects, this difference increases to 6 or $7 \mathrm{mag} \operatorname{arcsec}^{-2}$. The blue colors of LSB disks (Schombert et al. 1990) indicate that the differences between bulges and disks relates to the method of star formation (spacing, metallicity, IMF, etc. ...) and not the history of their stellar populations (e.g., fading).

The amount and spatial distribution of star formation can be seen in the few $\mathrm{H} \alpha$ images for F415-3, F469-2, F530-3, F614-1, and F746-1. By definition, LSB galaxies do not have high star formation rates, as measured by the number of massive stars per unit disk area. The $\mathrm{H} \alpha$ images demonstrate that the star formation is weak and sporadic. The few $\mathbf{H}$ II regions are widely spaced with little or no pattern and the total fluxes are low. The reader should compare these images to the image of M101 in $\mathrm{H} \alpha$ to note that a HSB galaxy's disk is consumed with star formation. The $\mathrm{H}$ II regions in LSB galaxies are associated with weakly enhanced regions of sur- 


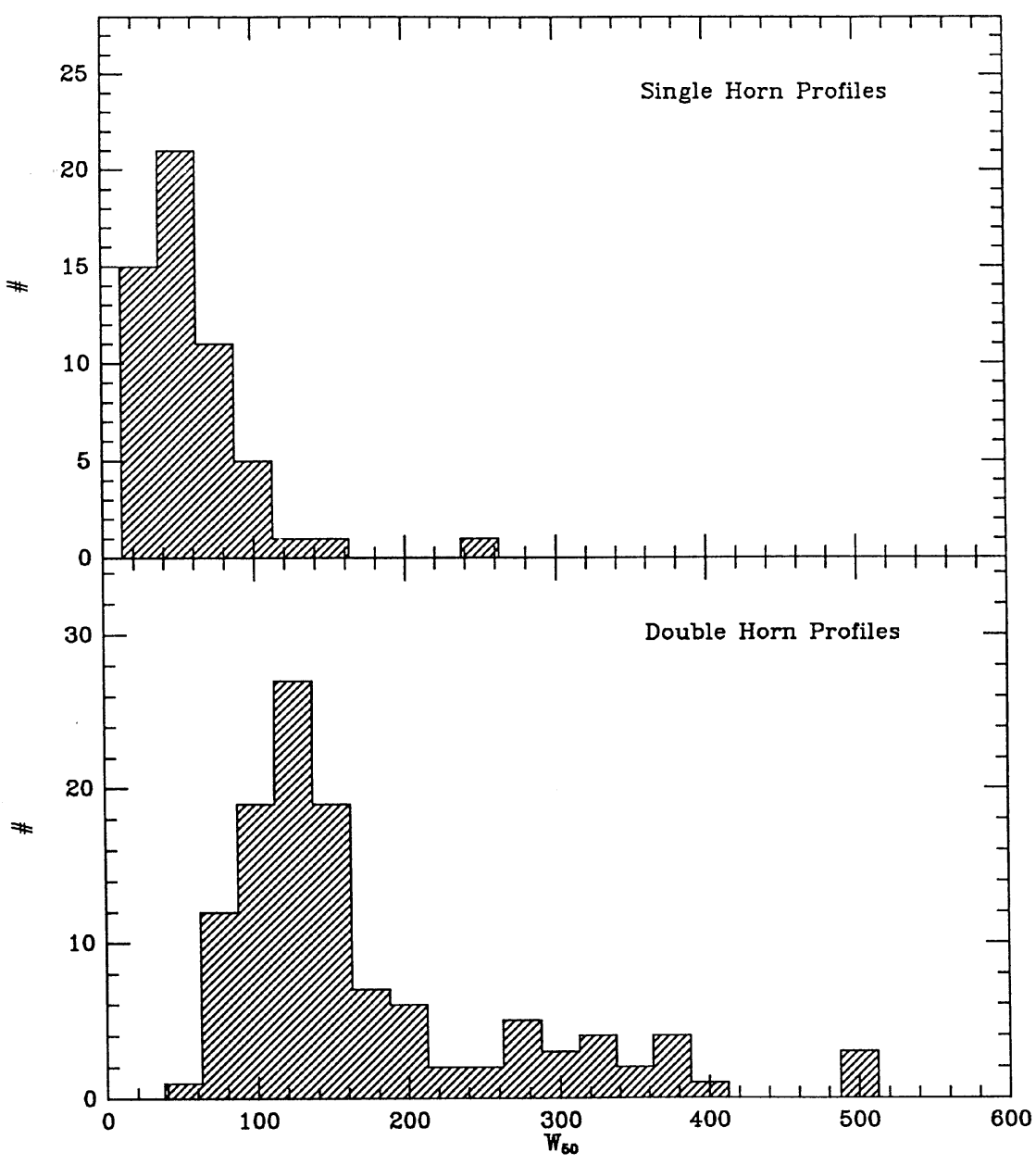

FIG. 6. $\mathrm{H}$ I velocity width in $\mathrm{km} \mathrm{s}^{-1}$ at $50 \%$ peak flux. The bottom panel displays the distribution of $W_{50}$ for objects identified as double horned. The upper panel displays $W_{50}$ for Gaussian profiles.

face brightness, but by no means the bright $\mathrm{OB}$ associations typical in M101. This has led us to speculate on the internal nature of the star formation in LSB galaxies, in particular the IMF may be skewed so that the formation of high-mass stars relative to the low-mass end of the main sequence is suppressed (Schombert et al. 1990). A fuller report on stellar population studies and metallicity measurements of the $\mathrm{H}$ II regions will occur at a later date. However, initial data gathered by McGaugh et al. (1991) indicate that abundances of $5 \%-10 \%$ solar are observed in $\mathrm{H}$ II regions in some LSB disks whose total luminosity is two to five times that of the Milky Way.

\section{SUMMARY}

This catalog attempts to extend our recognition of, and hopefully thereby our understanding of, a realm of galaxies close to the brightness of the sky. This region of observational parameter space was long thought to be dominated by small, dwarf galaxies whose low densities and faded stellar populations placed them far from the attributes of the HSB, so-called "normal" galaxies. In fact, the LSB domain is occupied by as full a spectrum of types as is encompassed by the Hubble sequence, and studies of the LSB galaxies may illuminate our understanding of the formation and star forma- tion history of galaxies in general. Our observations are summarized as the following:

(1) LSB disk galaxies are not dwarf versions of HSB spirals. They have similar sizes and màsses as HSB counterparts. The redshift distribution of LSB galaxies shows that a similar range of galaxy sizes is discovered in this catalog as compared to the UGC. Increased survey depth in surface brightness space is a process of recovering dwarfs, LSB disks and Malin objects. Only this last type is inherently new, not in terms of Hubble type, but in terms of scale length. Thus, a LSB survey recovers both small and very large galaxies.

(2) Ellipticals and S0's are rare in a LSB survey. The earliest types are mostly E's with extended envelopes (cD or shell types ). LSB S0's are similar in style with early type LSB spirals in that their disks are low in central surface brightness compared to their bulges. Their rarity in this survey may indicate a difference in the global style of evolution between S0 and spiral disks.

(3) Many of the LSB spirals have stellar-like nuclei (see F530-1 and F558-1 in Fig. 8). It is possible that many of the LSB disks have the same high occurrence of AGN phenomenon as the Malin objects have shown (Impey \& Bothun 1989).

(4) A majority of the LSB catalog is composed of late type galaxies in the Sc, Sm, and Im class. As the mean surface 


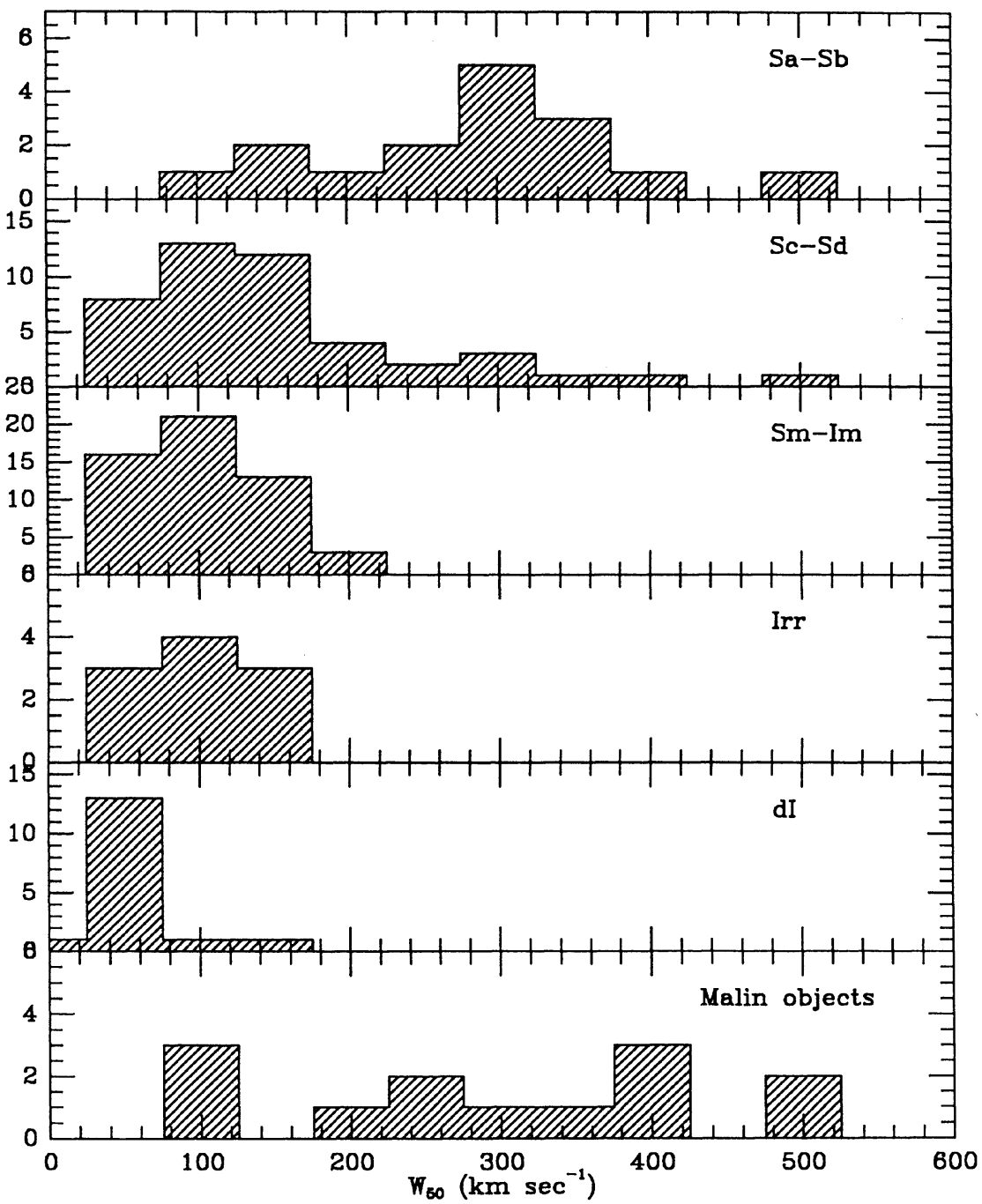

FIG. 7. $\mathrm{H}$ I velocity width in $\mathrm{km} \mathrm{s}^{-1}$ at $50 \%$ peak flux for each morphological class. The high-velocity Malin objects have a similar distribution as compared to early type spirals. No inclination corrections have been made. brightness of these galaxies decreases, a confident classification as either $\mathrm{Sm}, \mathrm{Im}, \mathrm{dI}$, or $\mathrm{dE}$ becomes impossible.

(5) $\mathrm{H}$ I measurements are presented for 171 objects. To the limits of the Arecibo velocity range, 34 nondetections are found. This gives a success rate of $80 \%$ for a LSB sampled catalog. We cannot rule out the possibility that the majority of the undetected galaxies lie at redshifts larger than the limit of our $\mathrm{H}$ i survey.

(6) The redshift distribution of this catalog is similar to the distribution of the UGC (another angular diameter limited catalog) with only an enhanced number of distant objects to differentiate the LSB sample (Malin objects). LSB galaxies are good tracers of large-scale structure on scales $>5 \mathrm{~h}^{-1} \mathrm{Mpc}$ but do avoid cluster cores. There is no evidence from this survey that LSB galaxies fill voids.

(7) The distribution of $\mathrm{H}$ I masses for this sample is not significantly different from the UGC. This implies that $\mathrm{H}_{\mathrm{I}}$ selection may recover a fuller range of galaxy types than traditional optical selection. LSB galaxies have a significant space density but, unless there are vastly more objects below our sensitivity threshold, their discovery does not appreciably alter $\Omega_{\text {baryon }}$.
(8) Broadband and $\mathrm{H} \alpha$ images of selected galaxies extend our impression of star formation over a larger surface brightness baseline. Star formation, as mapped by $\mathrm{H}$ II regions, is weak and spotty unlike either grand design or fragmented star formation styles in HSB galaxies. In fact any star formation is surprising in light of the lack of either molecular gas or enhanced surface brightness regions (see Schombert et al. 1990). Hence, star formation in LSB disks may offer us a window into the first epoch of star formation in all disk galaxies.

The most rewarding aspect of our survey is not simply an increased listing of the number of known galaxies, but the increase in the number of objects available to study a new realm of galaxy properties. A study of LSB galaxies is also not simply a study of dwarf galaxies, but one where star formation has acted several orders of magnitude lower than in HSB galaxies. Several extrapolated past histories exist for these types of galaxies and exploring them could provide possible links to phenomenon at high redshifts such as the large number of blue galaxies (Tyson 1988) or QSO absorption lines (York et al. 1986). Indeed, the existence of LSB disk galaxies in the nearby Universe may be related to rapid 


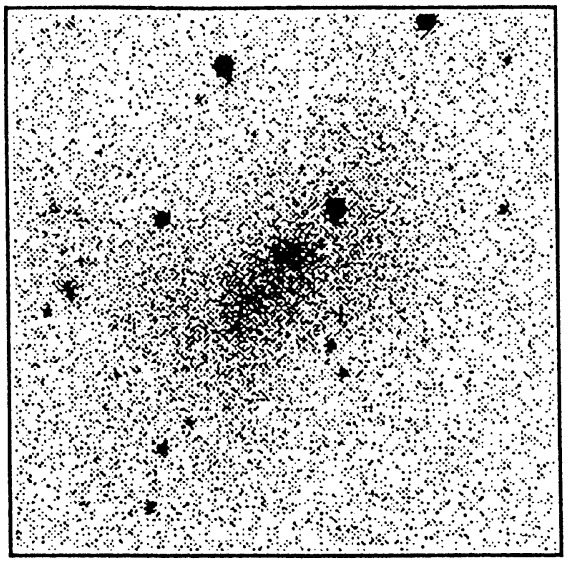

F415-3 R

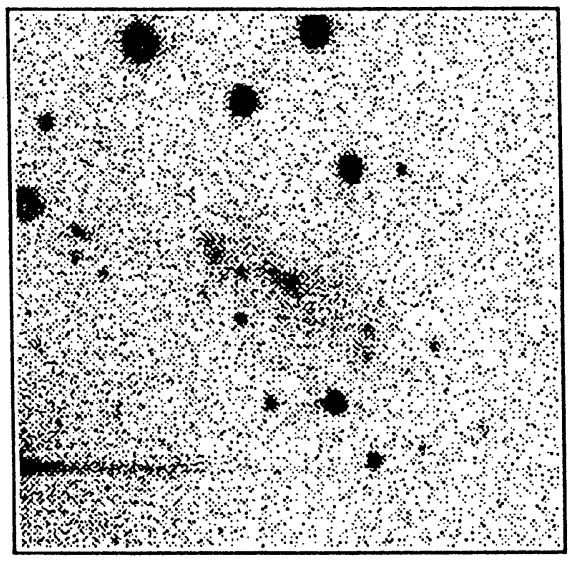

F469-2 R

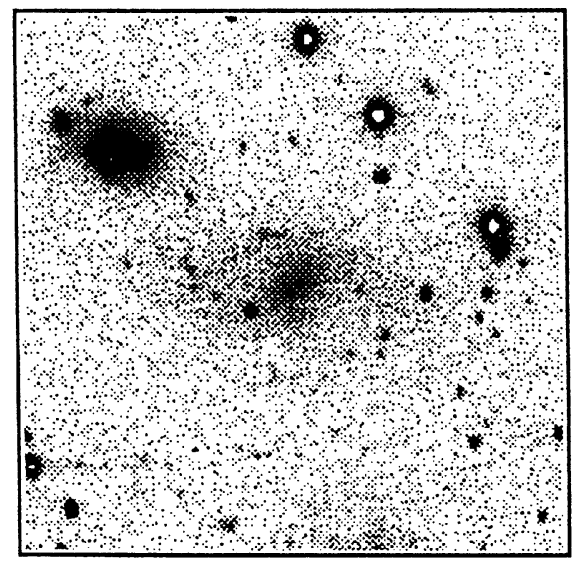

F530-3 R

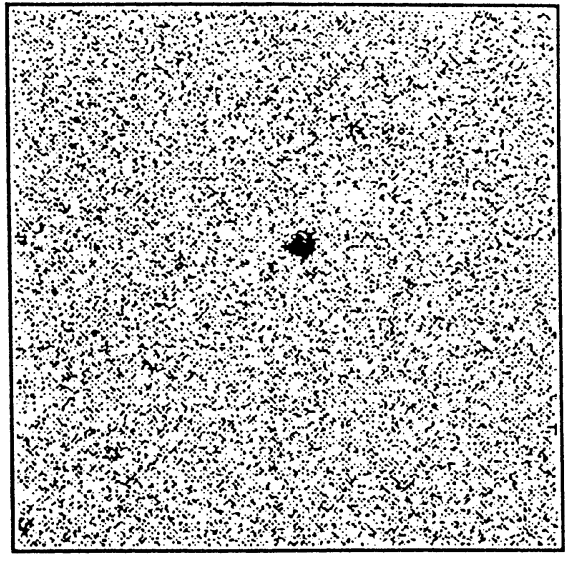

F415-3 H-alpha

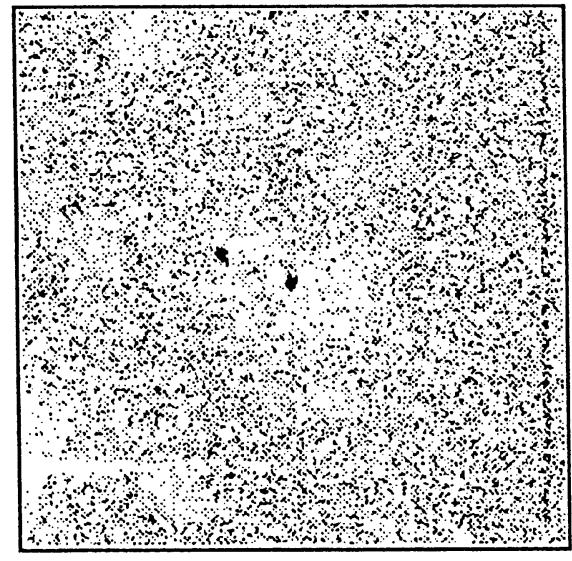

F469-2 H-alpha

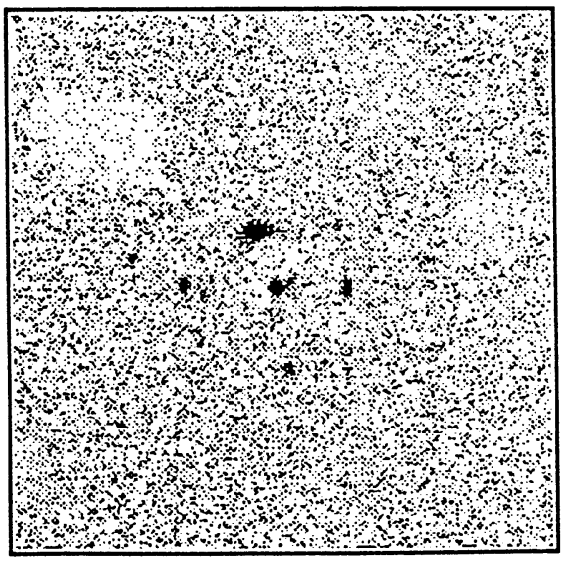

F530-3 H-alpha
FIG. 8. Optical CCD images for the LSB catalog. See text for discussion. 


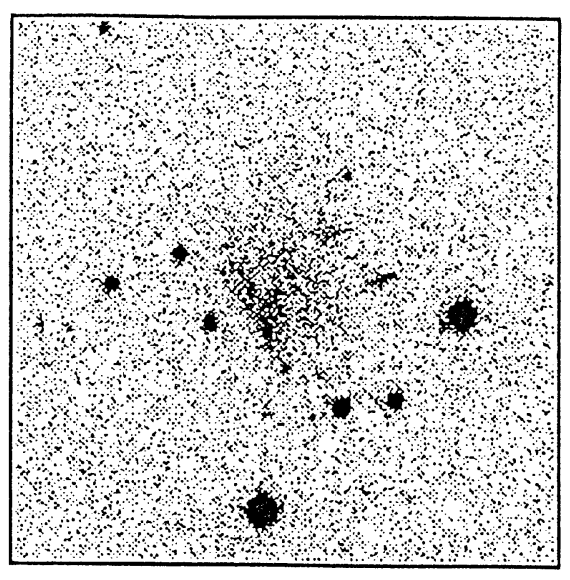

F614-1R

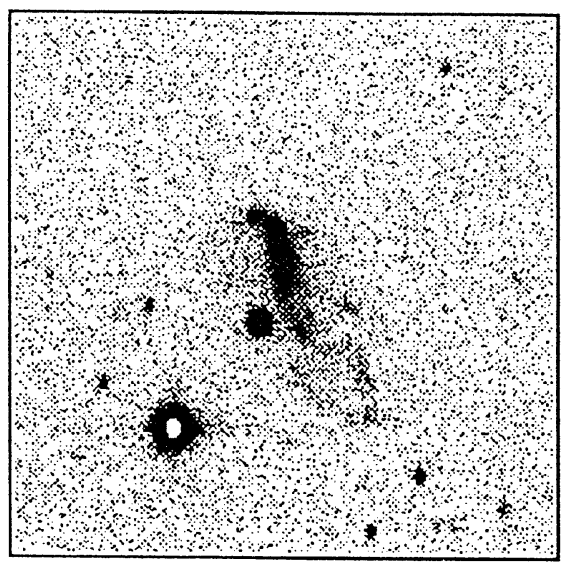

F746-1R

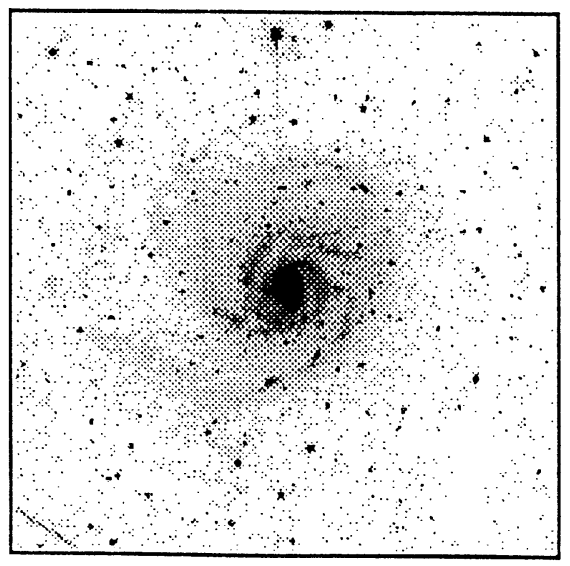

M101 R

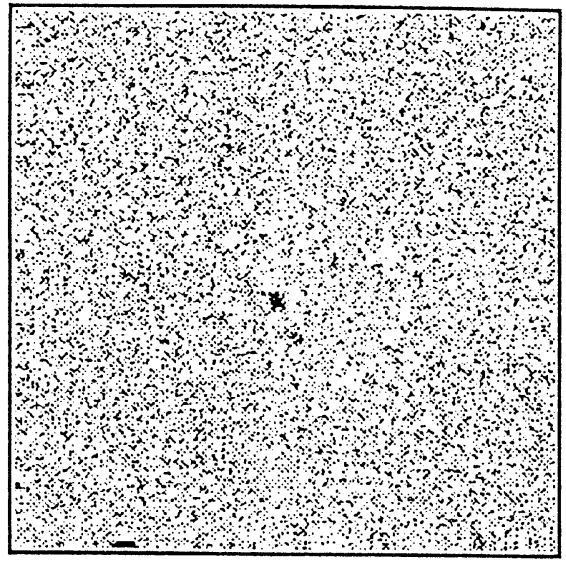

F614-1 H-alpha

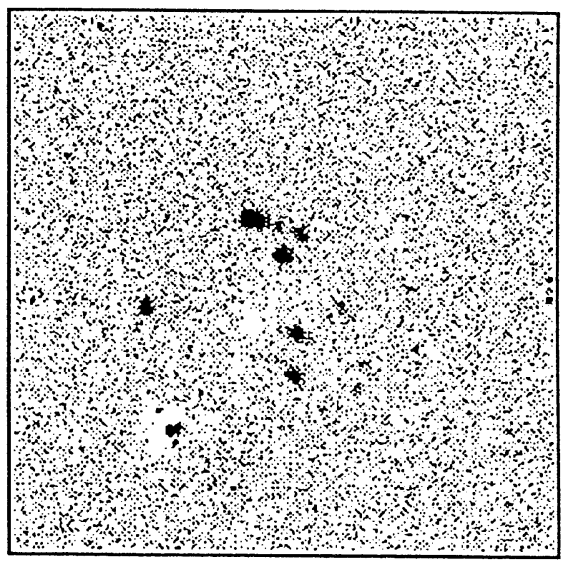

F746-1 H-alpha

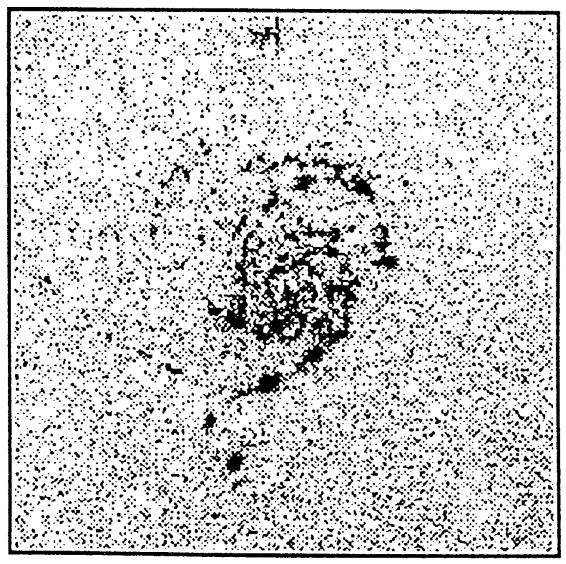

M101 H-alpha

FIG. 8. (continued) 


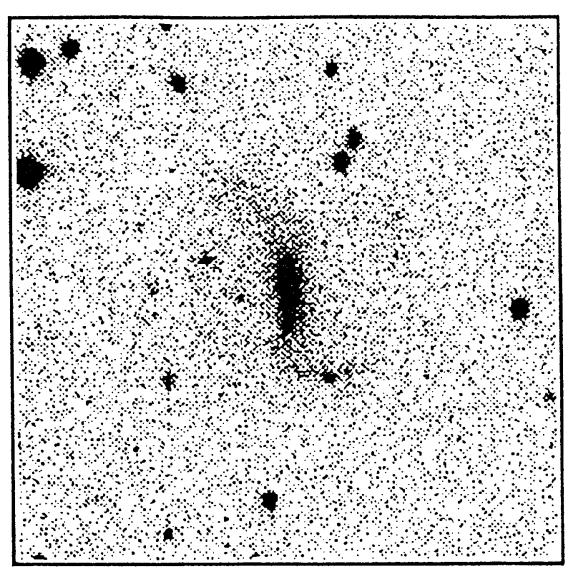

F480-1 R

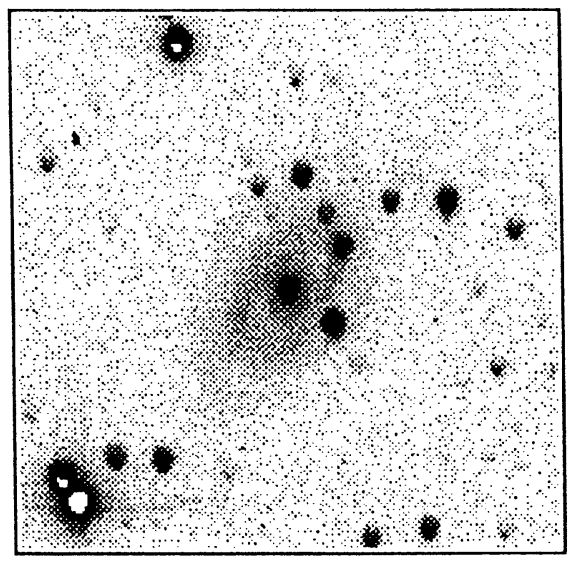

F530-1R

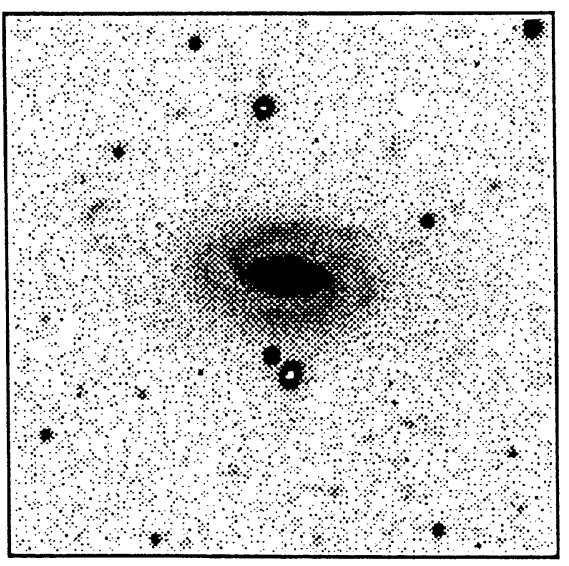

F533-3 R

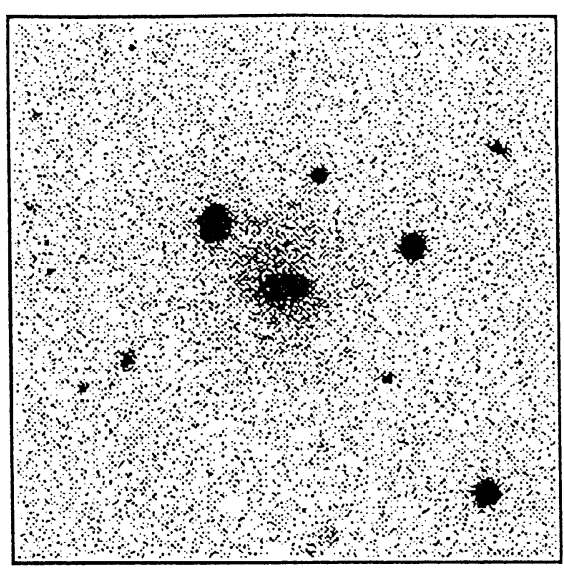

F480-V4 R

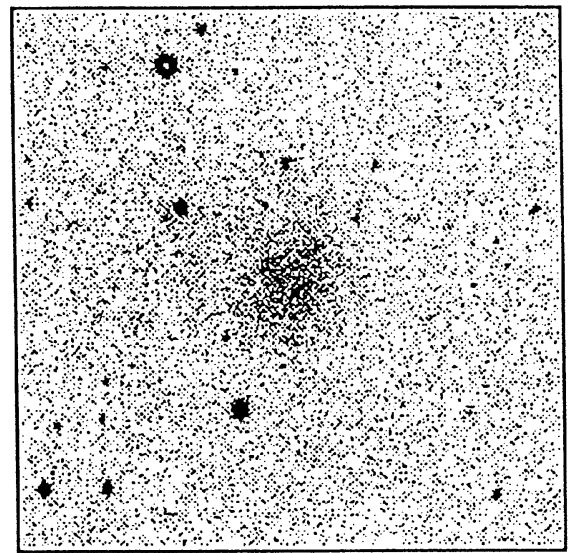

F533-1R

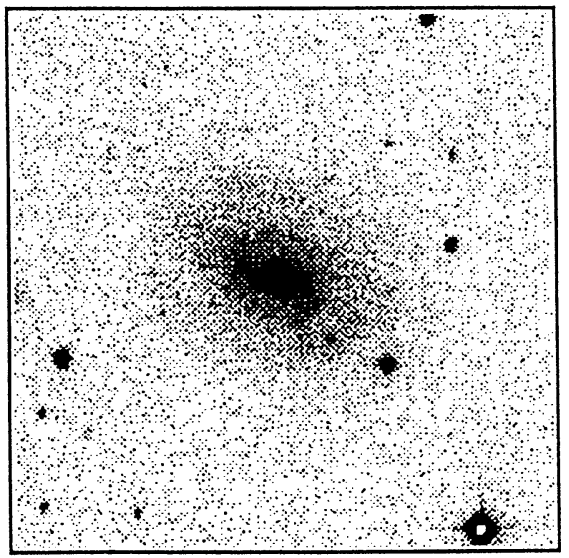

F539-2 R

FIG. 8. (continued) 


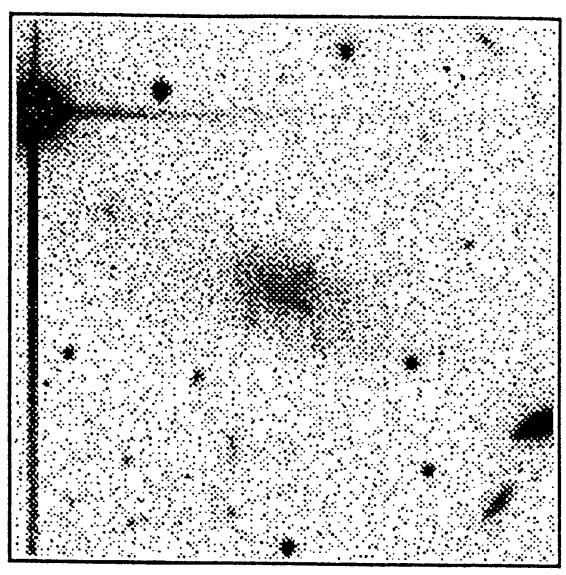

F544-1R

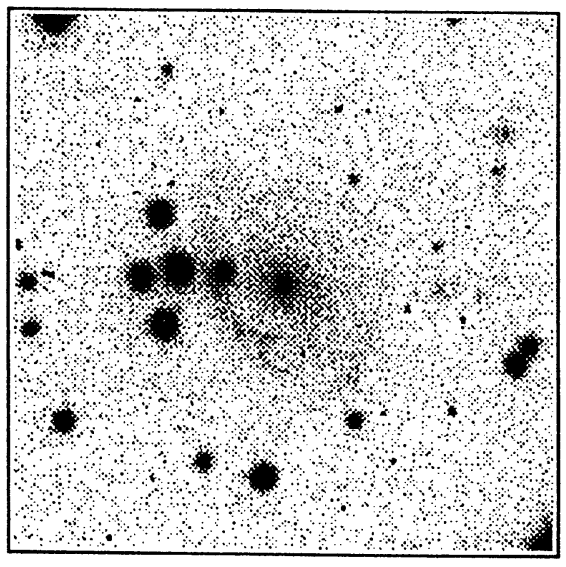

F558-1 B

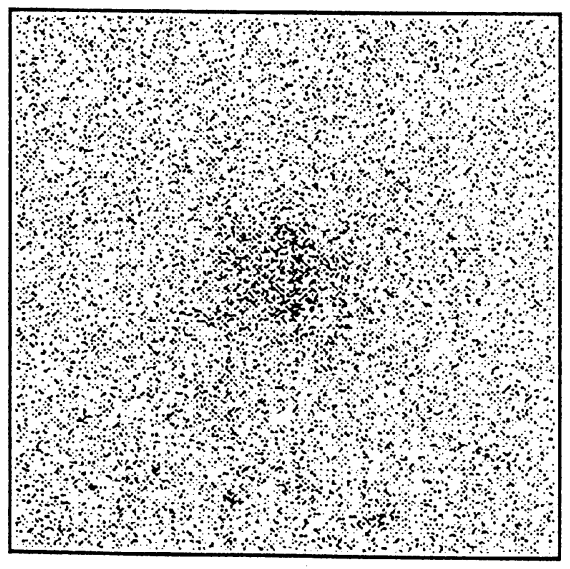

F564-V3 V

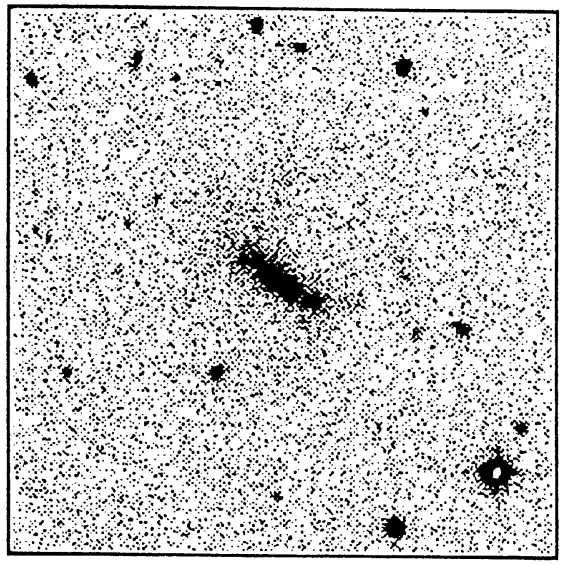

F544-2 R

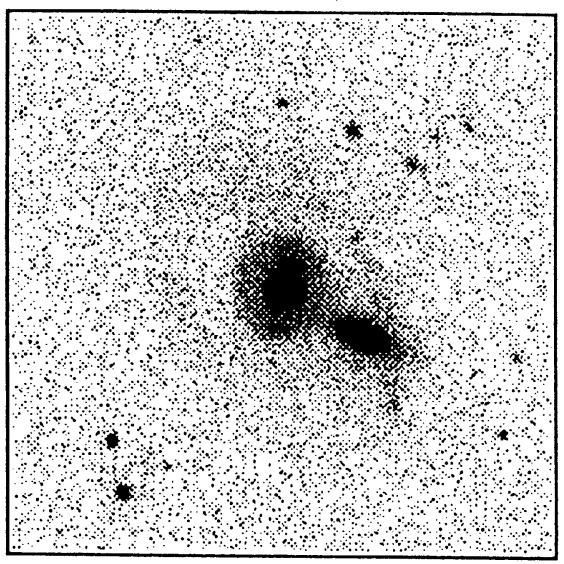

F564-1 V

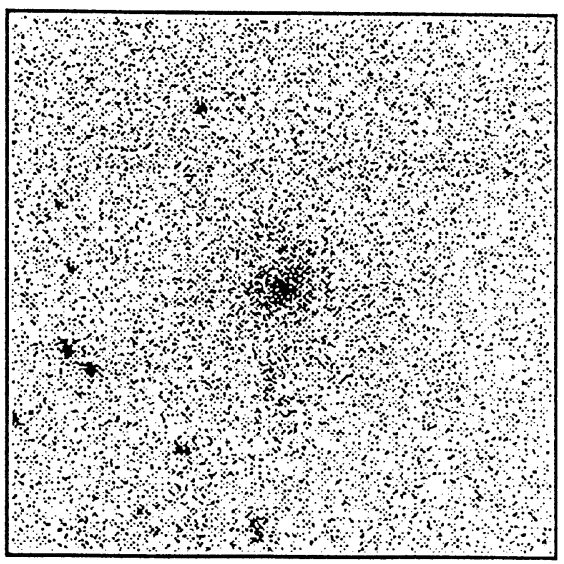

F567-2 V

FIG. 8. (continued) 


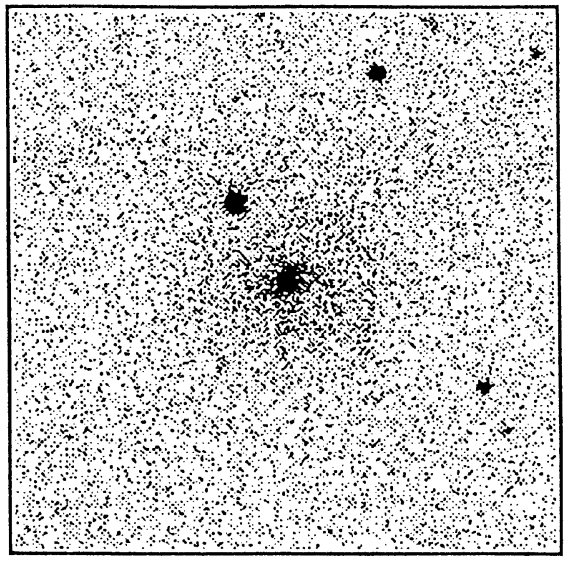

F568-1 V

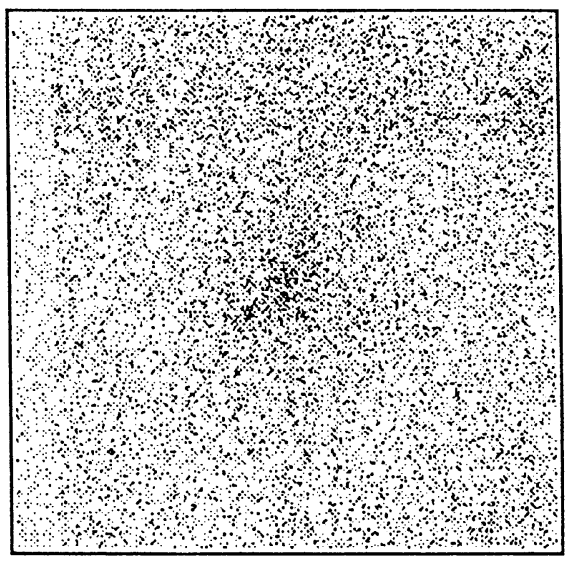

F574-2 V

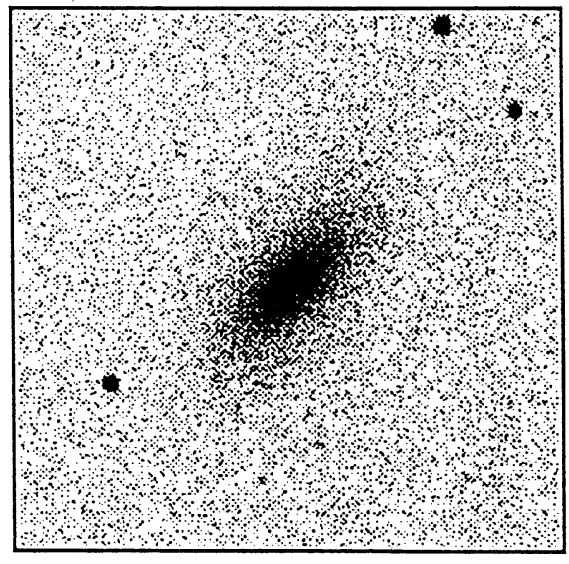

F574-7 V

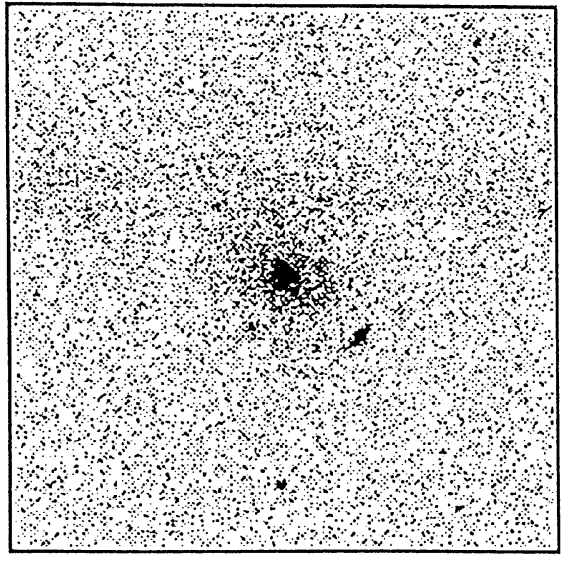

F568-V1 V

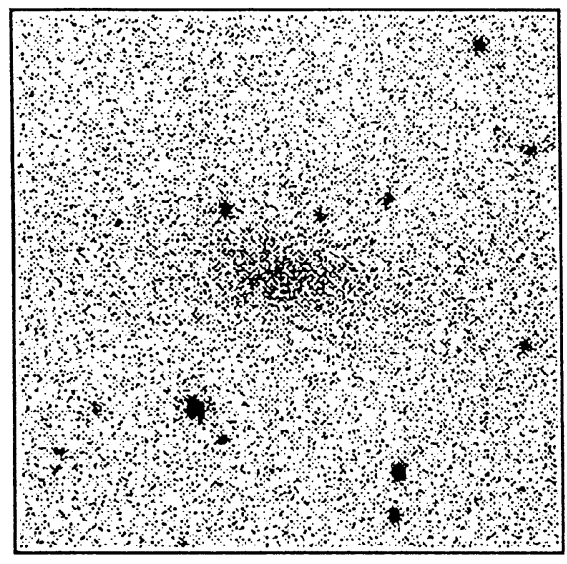

F574-6 V

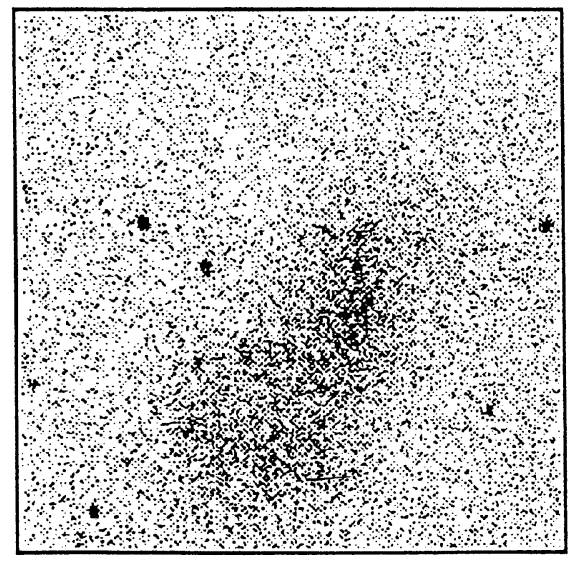

F575-3 V

FIG. 8. (continued) 


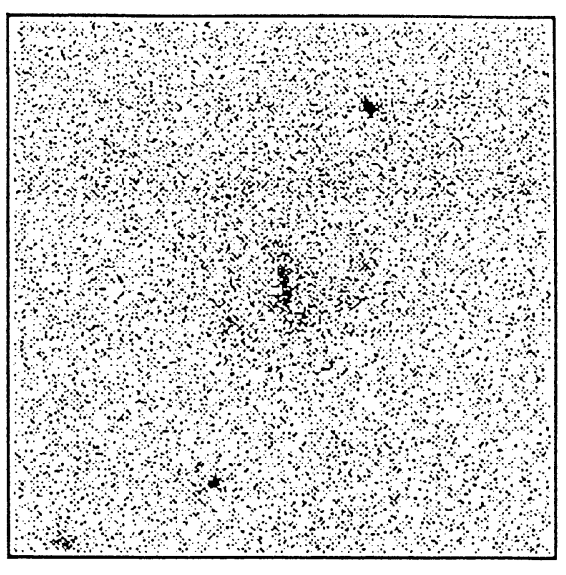

F577-V1 V

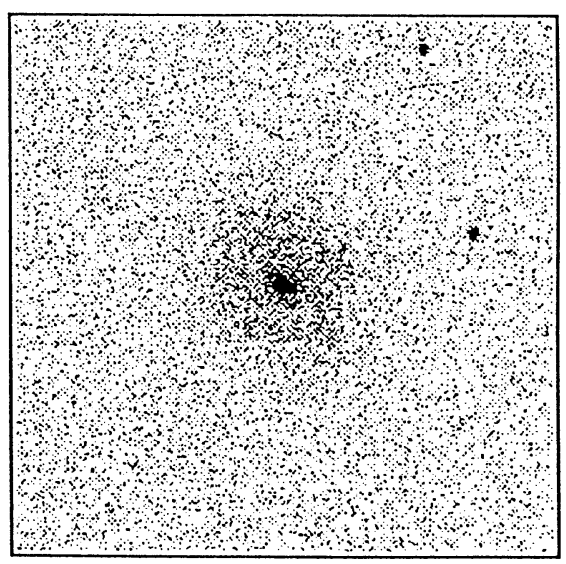

F579-V1 V

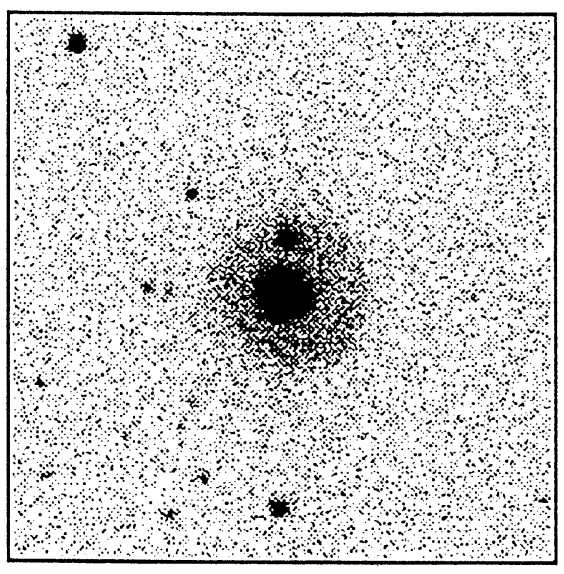

F582-V1 V

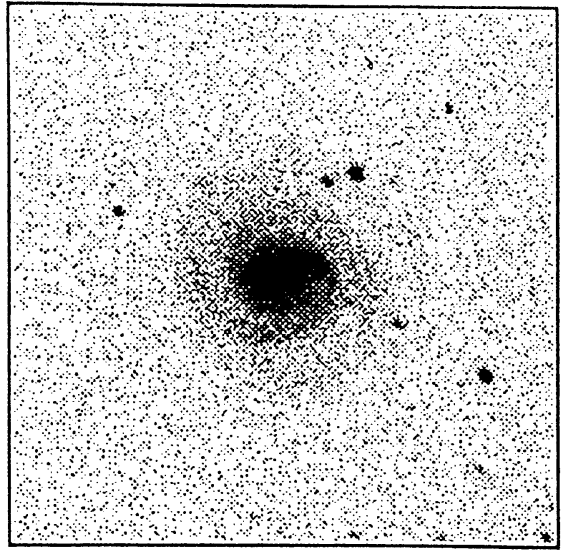

F578-2 V

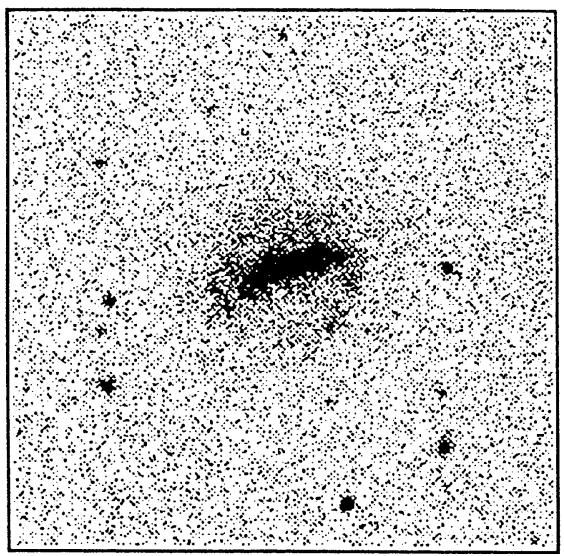

F581-V1 V

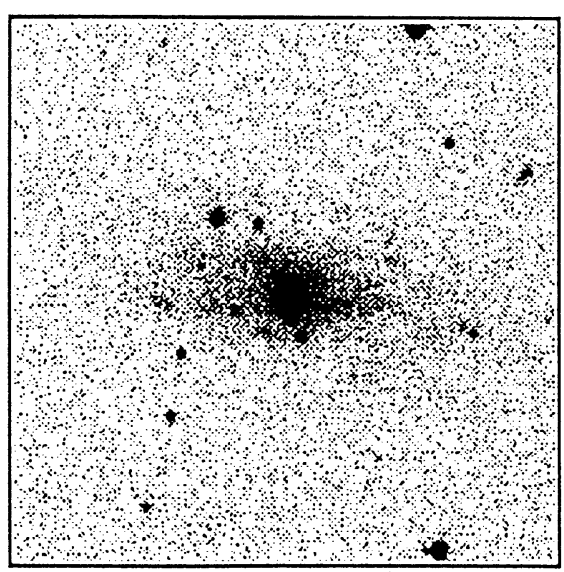

F583-1 V

FIG. 8. (continued) 


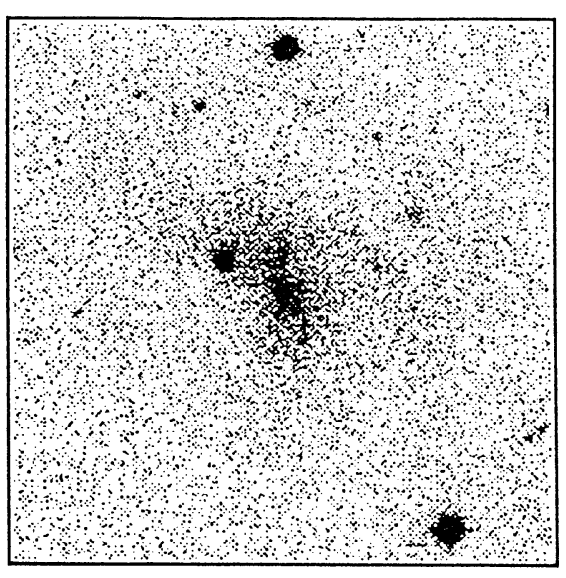

F583-2 V

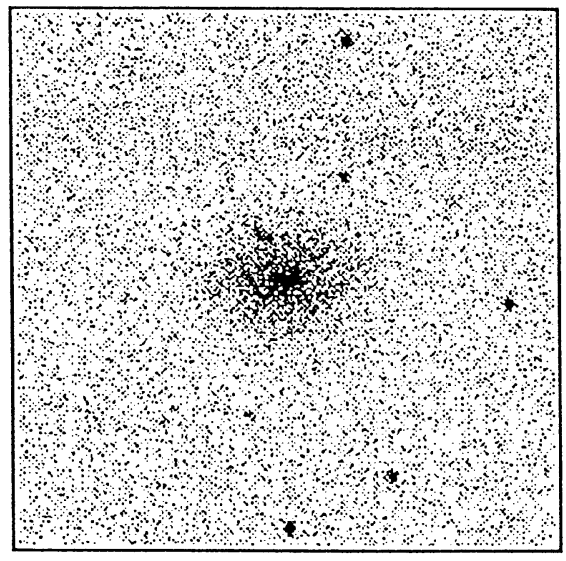

F583-V1 V

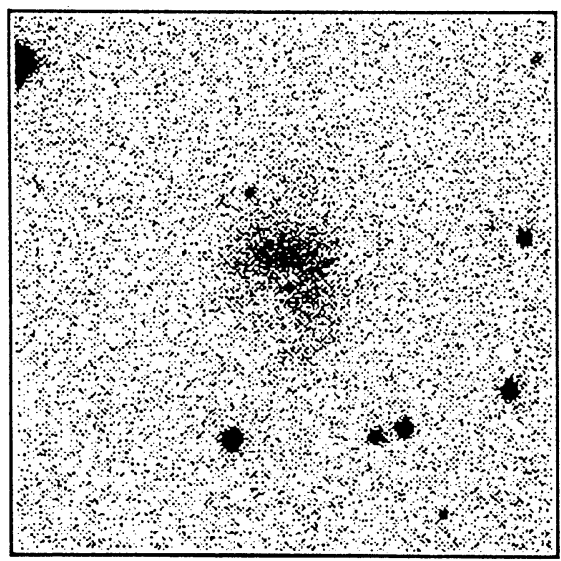

F585-V1 V

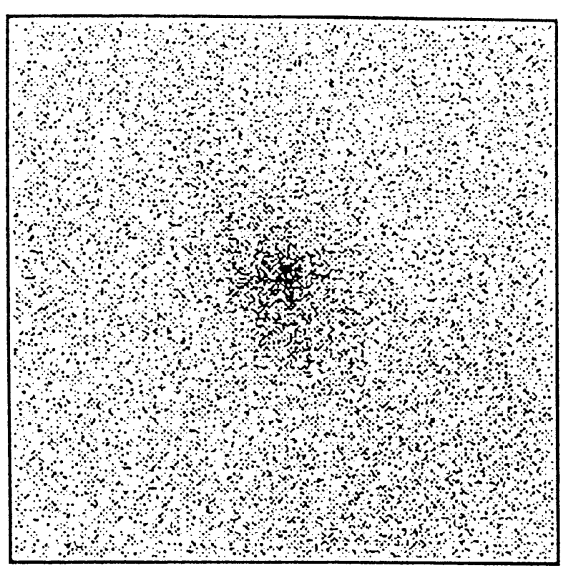

F583-4 V

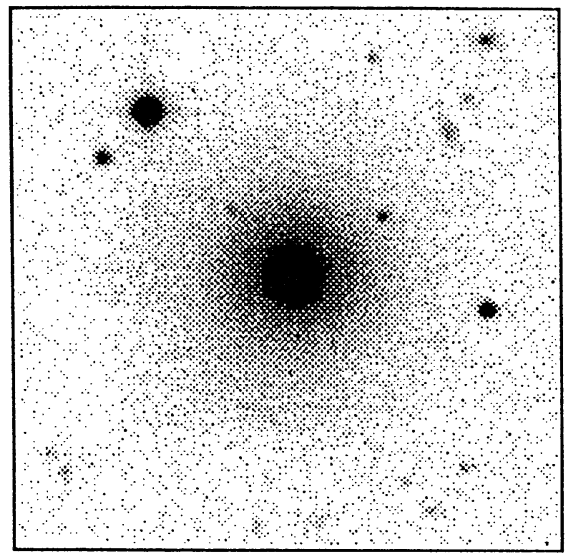

F585-1 V

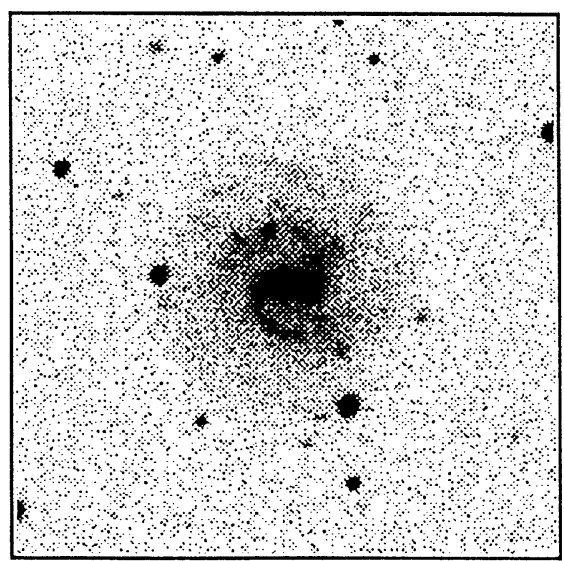

F602-1 R

FIG. 8. (continued) 


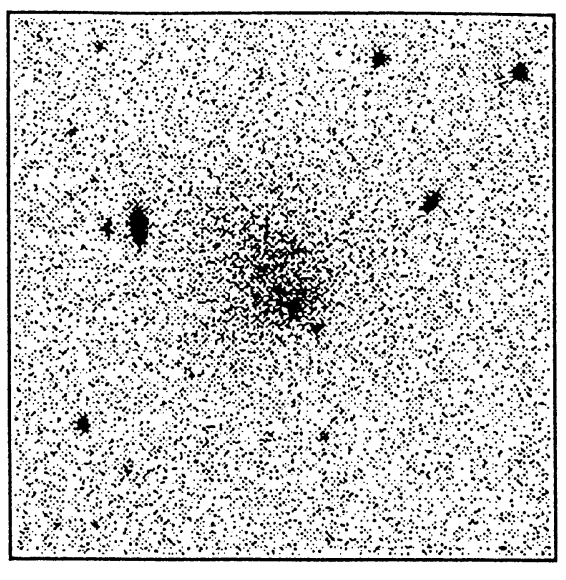

F611-1R

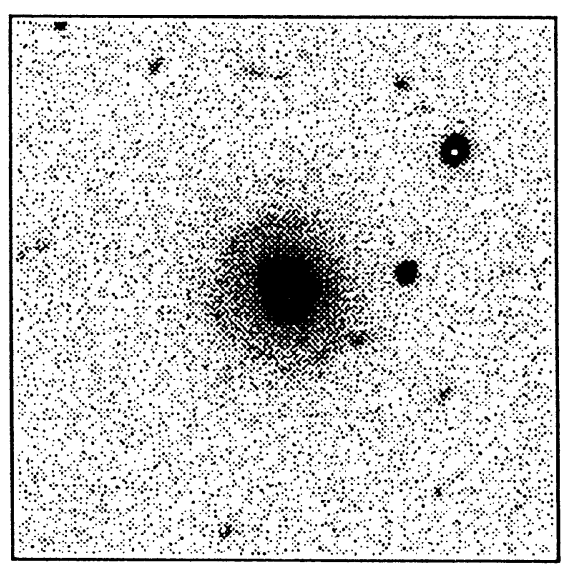

F612-V2 R

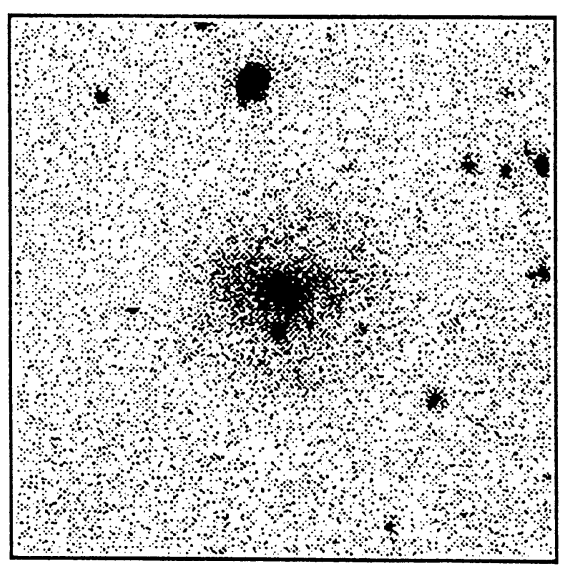

F687-2 R

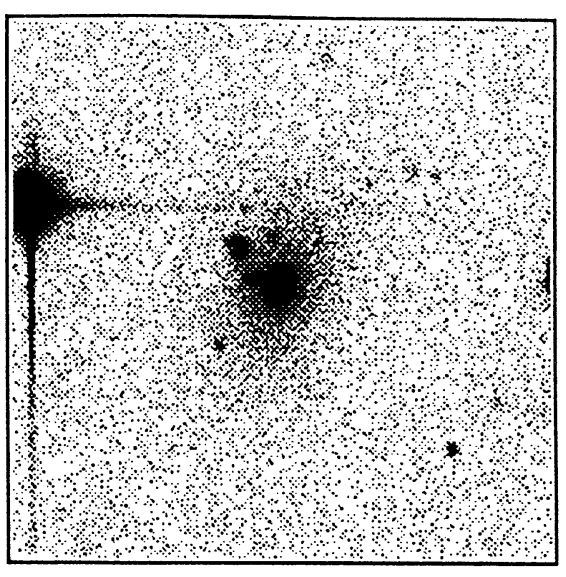

F611-3 R

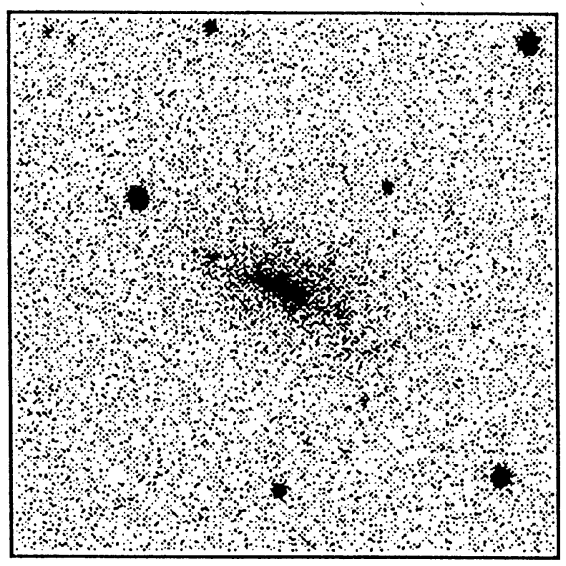

F677-3 R

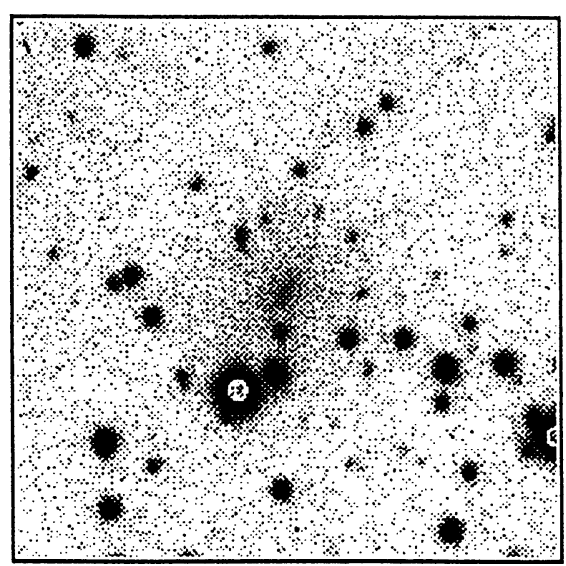

F740-1 R
FIG. 8. (continued) 
evolution of some component of the galaxy population at high redshift. In this case, such galaxies would only have strong contrast with respect to the background sky for a limited period of time. Over the majority of their existence they would simply have been missed.

The authors wish to acknowledge the Second Palomar Sky Survey team, both at Caltech and on Palomar Mountain: W. Sargent, G. Neugebauer, J. Mould, I. N. Reid, J. Muller, J. Phinney, A. Maury, B. Brucato, and R. Thicksten. This work is based on photographic plates obtained at Palomar Observatory 48 in. Oschin Telescope for the Second Palomar Sky Survey which was funded by the Eastman Kodak Company, the National Geographic Society, the
Samuel Oschin Foundation, the Alfred Sloan Foundation, the National Science Foundation Grant Nos. AST84-08225 and AST87-19465, and the National Aeronautics and Space Administration Grant Nos. NGL 05002140 and NAGW 1710. The research described in this paper was carried out by the Jet Propulsion Laboratory, California Institute of Technology, and was sponsored by the National Aeronautics and Space Administration. JS also wishes to thank A. Sandage for his timely observation that many of the early type spirals in this catalog have stellar-like nuclei. We also thank J. Gallagher for the CCD frame of M101 and J. Salzer and P. Knezek for the redshift distribution software and discussions. Many of the CCD frames taken for Fig. 8 were produced with a filter set constructed from the Theodore Dunham, Jr. Grant of the Fund for Astrophysical Research.

\section{REFERENCES}

Arp, H. C., \& Madore, B. F. 1987, A Catalogue of Southern Peculiar Galaxies and Associations (Cambridge University Press, Cambridge)

Binggeli, B., Tarenghi, M., \& Sandage, A. 1991, preprint

Bothun, G. D., Beers, T. C., Mould, J. R., \& Huchra, J. P. 1986, ApJ, 308, 510

Bothun, G. D., Impey, C., Malin, D., \& Mould, J. R. 1987, AJ, 94, 23

Bothun, G. D., Impey, C., \& Malin, D. 1991, ApJ, 376, 404

Bothun, G. D. 1990, in Evolution of the Universe, edited by R. Kron (Astronomical Society of the Pacific, San Francisco), p. 54

Bothun, G. D., Schombert, J. M., Impey, C. D., \& Schneider, S. E. 1990 ApJ, 360, 427

Cornell, M., Aaronson, M., Bothun, G. D., \& Mould, J. 1987, ApJS, 64, 507

Dressler, A. 1980, ApJ, 236, 351

Freeman, K. 1970, AJ, 160, 811

Helou, G, Madore, B. F., Schmitz, M., Bicay, M. D., Wu, X., \& Bennett, J. 1991, in Databases and On-Line Data in Astronomy, edited by D. Egret and M. Albrecht, (Kluwer, Dordrecht)

Hodge, P. W., Pyper, D. M., \& Webb, C. W. 1965, AJ, 70, 559

Huchtmeier, W. K., \& Richter, O. G. 1983, A General Catalog of H I Observations of Galaxies (Springer, New York)

Impey, C., Bothun, G. D., \& Malin, D. F. 1988, ApJ, 330, 634

Impey, C., \& Bothun, G. D. 1989, ApJ, 341, 89

Impey, C., Bothun, G. D., \& Malin, D. 1992, in preparation

de Lapparent, V., Geller, M. J., \& Huchra, J. P. 1986, ApJl, 302, L1

McGaugh, S., Bothun, G. D., \& Schombert, J. M. 1991, in preparation

Malin, D. F., \& Carter, D. 1980, Nature, 285, 643

Nilson, P. 1973, Uppsala General Catalogue of Galaxies, Uppsala Astron.
Obs. Ann., 6

Postman, M., \& Geller, M. J. 1984, ApJ, 281, 95

Reid, I. N., et al. 1991, PASP, 103, 661

Salzer, J. J., Alighieri, S. S., Matteucci, F., Giovanelli, R., \& Haynes, M. P. 1991, AJ, 101, 1258

Sandage, A., \& Tammann, G. A. 1981, Revised Shapley-Ames Catalog of Bright Galaxies (Carnegie Institution of Washington Publication No. 635)

Sandage, A., \& Binggeli, B. 1984, AJ, 89, 919

Schneider, S. E., Thuan, T. X., Magri, C., \& Wadiak, J. E. 1990, ApJS, 72 245

Schombert, J. M., \& Bothun, G. D. 1988, AJ, 95, 1389

Schombert, J. M., Bothun, G. D., Impey, C. D., \& Mundy, L. G. 1990, AJ, 100,1523

Silk, J. 1991, IAU Colloquium No. 149, in preparation

Smith, H. E., Cohen, R. D., Burns, J. E., Moore, D. J., \& Uchida, B. J. 1989, ApJ, 347, 87

Thuan, T. X., Alimi, J., Gott, J. R., \& Schneider, S. E. 1991, ApJ, 370, 25

Tyson, J. A. 1988, AJ, 96, 1

de Vaucouleurs, G. 1959, in Handbuch der Physik, edited by S. Flugge, Vol. 53 , p. 275

van der Hulst, J. M., Skillman, E. D., Kennicutt, R. C., \& Bothun, G. D. 1987, A\&A, 177, 63

Wolfe, A. M., Turnshek, D. A., Smith, H. E., \& Cohen, R. D. 1986, ApJS, 61,249

York, D. G., Dopita, M., Green, R., \& Bechtold, J. 1986, ApJ, 311, 610 